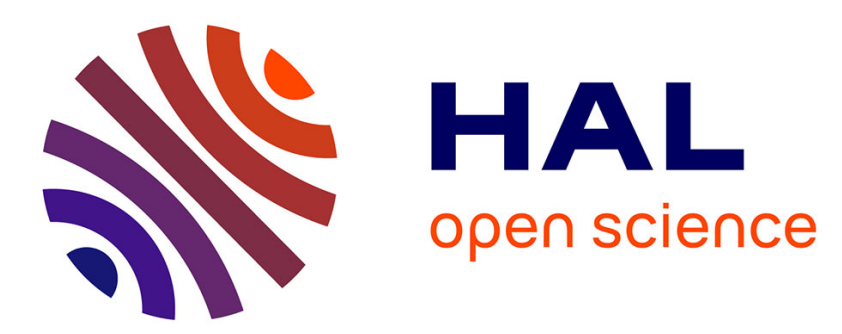

\title{
Hyperconnections and Hierarchical Representations for Grayscale and Multiband Image Processing
} Benjamin Perret, Sébastien Lefèvre, Christophe Collet, Éric Slezak

\section{To cite this version:}

Benjamin Perret, Sébastien Lefèvre, Christophe Collet, Éric Slezak. Hyperconnections and Hierarchical Representations for Grayscale and Multiband Image Processing. IEEE Transactions on Image Processing, 2012, 21 (1), pp.14-27. 10.1109/TIP.2011.2161322 . hal-00737998

\section{HAL Id: hal-00737998 https://hal.science/hal-00737998}

Submitted on 3 Oct 2012

HAL is a multi-disciplinary open access archive for the deposit and dissemination of scientific research documents, whether they are published or not. The documents may come from teaching and research institutions in France or abroad, or from public or private research centers.
L'archive ouverte pluridisciplinaire HAL, est destinée au dépôt et à la diffusion de documents scientifiques de niveau recherche, publiés ou non, émanant des établissements d'enseignement et de recherche français ou étrangers, des laboratoires publics ou privés. 


\title{
Hyperconnections and Hierarchical Representations for Grayscale and Multiband Image Processing
}

\author{
Benjamin Perret*, Sébastien Lefèvre, Christophe Collet, and Éric Slezak
}

\begin{abstract}
Connections in image processing are an important notion that describes how pixels can be grouped together according to their spatial relationships and/or their gray level values. In recent years, several works were devoted to the development of new theories of connections among which hyper-connection (h-connection) is a very promising notion. This paper addresses two major issues of this theory. First, we propose a new axiomatic which ensures that every h-connection generates decompositions that are consistent for image processing and more precisely for the design of $\mathrm{h}$-connected filters. Second, we develop a general framework to represent the decomposition of an image into h-connections as a tree which corresponds to the generalization of the connected component tree. Such trees are indeed an efficient and intuitive way to design attribute filters or to perform detection tasks based on qualitative or quantitative attributes. These theoretical developments are applied to a particular fuzzy h-connection and we test this new framework on several classical applications in image processing: segmentation, connected filtering, and document image binarization. The experiments confirm the suitability of the proposed approach: it is robust to noise and it provides an efficient framework to design selective filters.
\end{abstract}

Index Terms-Edics: SMR-STM, Hyperconnection, hierarchical representation, connected operator, connected filter, document image binarization, mathematical morphology, connection, Max-Tree, image filtering, image segmentation.

\section{INTRODUCTION}

$\mathrm{I}$ N DIGITAL IMAGE PROCESSING, the notion of connection describes how pixels are grouped together to form objects called connected components. At the very beginning, there were two distinct ways to perform such grouping: the topological connection (where a set is connected if it cannot be partitioned into two disjoint open sets) and the path-connectivity (where the pixels of the image are considered as nodes of a graph and a set is connected if a path of edges exists between all nodes). The notion of set connections was introduced in the late 1980s by Serra [1] as a theory to unify these previous approaches into a common algebraic framework. In this theory, a connection is composed of all connected sets and the connected components of an image are then the greatest connected sets included in this image. Thus

- Copyright (c) 2010 IEEE. Personal use of this material is permitted. However, permission to use this material for any other purposes must be obtained from the IEEE by sending a request to pubs-permissions@ieee.org.

- Benjamin Perret, and Christophe Collet are with the Image Science, Computer Science and Remote Sensing Laboratory (LSIIT, UMR 7005, University of Strasbourg-CNRS). Address: LSIIT, Pôle API, Bd Sébastien Brant, BP 10413, 67412 Illkirch Cedex France.

E-mails: \{bperret,c.collet\}@unistra.fr

- Sébastien Lefevre is with the VALORIA Laboratory, University of South Brittany. Address: VALORIA Lab, Campus de Tohannic, BP 573, 56017 Vannes Cedex, France

E-mail: sebastien.lefevre@univ-ubs.fr

- Éric Slezak is with the University of Nice-Sophia Antipolis in the Cassiopée Laboratory (OCA, UMR 6202). Address: CNRS-Observatoire de la Côte d'Azur, Boulevard de l'Observatoire, BP 4229, 06304 Nice, Cedex 4, France.

E-mail:eric.slezak@oca.eu different connections will lead to different divisions of the image into connected components. The notion of connection has had a great impact on mathematical morphology and was followed by the introduction of connected operators in binary images [2] and grayscale images [3], [4], [5]. These operators have the property to either preserve or delete the connected components of an image, and therefore do not modify object boundaries.

The connected operators, which are based on set connections, have been very successful in image filtering [4], [6], [7] or segmentation [8], [9], [10], [11], [12]. All these approaches rely on the original connection theory which is set oriented and the extension to grayscale images is realized through thresholding (i.e. flat-zone filtering). The threshold decomposition has the advantage of being conveniently represented by a tree structure [5] (known as component tree [13], Max-Tree [5], dendrone [14], or confinement tree [15]) leading naturally to the notion of hierarchical representation.

Nevertheless, the flat-zone approach can be affected by various problems:

- sensitivity to noise: a flat zone representing an object is easily broken by noise;

- linkage: different objects are grouped by artifacts or small features into the same flat zone;

- set connections and gray levels: underlying set connections can only take spatial relationships into account, thus do not consider gray levels which can cause an object with gray-level gradients or textures to be spread into several flat zones;

- overlapping: underlying set connections cannot 
handle overlapping.

Recent developments have been made in the connection theories, including sometimes subsequent modifications of the component tree, to try to solve these issues. Section 1.1 describes those developments and section 1.2 is dedicated to the contributions of the paper.

\subsection{Related Work}

In [16], the author proposed relaxing the set connection theory to allow for the creation of partial set connections and partial partitions. Partial connections are of great interest when dealing with segmentation methods that may leave part of the image unsegmented (e.g. boundaries in a watershed), but do not solve the other problems mentioned.

Another extension of set connections are second generation connections (see [17] and references therein) which are defined by the application of an operator over a primary connection. Second generation connections are themselves set connections which can be divided between contraction-based connectivities (where connected components of the primary connection may become disconnected in the second generation connection) and clustering-based connectivities (where disconnected components of the primary connection may become connected in the second generation connection). The authors of [18] developed a method to perform attribute filtering over the Max-Tree using a special second generation connection called mask based second generation connectivity. Second generation connectivity may solve the problem of broken flat zones (with clustering based connections) and the problem of linkage (with contraction-based connectivities). Nevertheless, second generation connectivities are not able to deal with overlapping.

On the other hand, nearly ten years after his first proposal, Serra published [19] a new definition of connections based on the theory of complete lattices which enables the consideration of both spatial relations and gray level values. This new definition was immediately followed (at the end of the same article) by the proposition of a relaxed definition called h-connection, but this idea was not further developed in the article. The latter permits one to define a large variety of connections over arbitrary lattices (including grayscale and multiband images) and is able to deal with overlapping. It has been the basis of several recent works. In [20], the authors presented a specific type of h-connection and proposed the notion of segmentation by similarity zones (further developed in [21]). The work in [22] defined a new hconnection based on the theory of fuzzy connectivity measures and developed the notion of fuzzy attribute filtering. In [23] and [24], the authors proposed to modify the reconstruction step of the traditional attribute filter with the Max-Tree by using a specific h-connection called the $k$-flat zones. Finally, in [25] and [26] the author started to explore the theoretical properties of h-connections and their links with the other definition of connections. All of this progress and success linked to the h-connection theory has led us to believe that it is a very promising theory which deserves further investigation.

\subsection{Contributions}

We address two major issues of h-connections:

1) we develop a new class of h-connections that strengthens the current theory and allows more general and interesting properties to be derived from it;

2) we propose a generic framework to generate hierarchical representations from h-connections in order to provide an efficient and intuitive way to design hyper-connected attribute filters.

The axiomatic of the current theory is problematic. It is based on three fundamental axioms which informally state that 1) the least element is hyperconnected (h-connected) 2) the points are h-connected and 3) the supremum of overlapping hyper-components (h-components) is connected. While axioms 1 and 2, which establish that minimal elements are h-connected, are easily admitted, the third axiom is more difficult to justify. It introduces the novel notion of overlapping as a decreasing predicate on the h-components which is intuitively satisfactory, but in practice, does not allow any new property to be derived from the theory. But its most important weakness is the lack of consistency of derived h-connected operators: the theory does not ensure that the deletion of an h-component of an image will effectively change the image (Proposition 1). The corollary of this observation is that deleted h-components of an image may reappear in the result.

We propose a new property that formalizes the following idea: the decomposition of an image in hcomponents must be necessary and sufficient to describe the image. We think that this property is fundamental for image processing as it implies that the decomposition into h-components completely describes the whole image (sufficient) and that none of its components is useless (necessary). These requirements enforce the consistency of the h-connected filters as they ensure that: 1) every deletion of image components will effectively modify the filtered image 2) a deleted component can not re-appear in the filtered image.

From a more formal point of view, the sufficiency and necessity conditions can be formulated like this: the supremum of the h-components of an image must be equal to the whole image (sufficient) and the supremum of a family strictly included in the family of hcomponents must be strictly included in the image (necessary). In the current theory, the sufficiency is provided by axioms 1 and 2 . To ensure the necessity, we propose three equivalent properties formulating this requirement from different points of views.

Next, based on these new properties, the notion of hierarchical representation is explored. The traditional component tree representation is based on a partial 
ordering of the flat zones of an image, but in the hconnection theory, h-components are by definition not comparable. Nevertheless, the connected openings of the $\mathrm{h}$-connection theory introduce the interesting notion of z-zones. The z-zones are particular regions where all points generate the same set of connected components and the entire image can be divided into such zones. We show that the z-zones can be partially ordered and give a sufficient condition to ensure that their Hasse diagram with respect to this new ordering is a tree. This tree is thus a possible generalization of the component tree to non flat-zones based on h-connections. This hcomponent tree can then be used in the same manner as the traditional component tree, following the traditional processing steps: tree construction, tree pruning, and finally reconstruction of the result [27].

As the h-component is just a representation of the decomposition of the image according to a particular h-connection, its properties are thus inherited from the chosen h-connection and they cannot be specified until such an h-connection has been chosen.

These theoretical developments are illustrated by their application on a fuzzy h-connection recently proposed in [22]. Our new approach allows us to represent the different fuzzy h-connected components of an image in a single tree structure. This provides a convenient representation to design fuzzy h-connected operators and to reuse all existing algorithms imagined for the connected component tree in this new context. A simple and efficient algorithm is given to transform the component tree into the h-component tree of this h-connection. We first show how the component tree is simplified by the use of the h-connection. Then, we evaluate the performances of filtering operations on grayscale images compared to other connected filters. Next, we show that the method can be easily extended to multiband images. This extension is demonstrated on multispectral astronomical images of galaxies. Finally, we propose a novel document image binarization method based on the h-component tree and we evaluate it on the DIBCO (Document Image Binarization Contest [28]) 2009 benchmarking dataset.

\subsection{Organization}

In section 2, we go over some preliminary definitions about the lattice theory, the theory of set connections, and h-connections. Next, in section 3, we present the proposed evolution of the h-connection axiomatic. We give the different formulations of the proposed axioms and establish some new results. The theory of h-component trees is presented in section 4 . It includes a definition of the order relation between z-zones and the definition of the corresponding tree. Section 5 is dedicated to the applications. The fuzzy h-connection is first defined and a suitable algorithm is given to compute the h-component tree. Then, the experimental results are presented, we demonstrate the properties of the h-component tree on grayscale images, we show that it can handle multiband images, and we propose a new method for document image binarization. Finally, section 6 concludes the paper and suggests future research directions.

\section{Set Connection, lattice, and Hyper- CONNECTION}

In the sequel, sets and families are written in capital letters while elements of a set are written in lower-case letters. The logical conjunction (respectively disjunction) is noted $\wedge$ (respectively $\vee$ ). Proofs of the different propositions are given in the appendix.

\subsection{The Lattice Theory}

This section gives the necessary definitions on the lattice theory for this article, the reader may refer to [29], [30] for more information.

A lattice $\left(\mathcal{L}, \leq_{\mathcal{L}}, \bigvee_{\mathcal{L}}, \bigwedge_{\mathcal{L}}\right)$ is composed of a non-empty set $\mathcal{L}$ with a partial order $\leq_{\mathcal{L}}$ (a reflexive, transitive and antisymmetric binary relation) and two internal operators: a supremum $\bigvee_{\mathcal{L}}$ and an infimum $\bigwedge_{\mathcal{L}}$. In the sequel, when no confusion is possible, we drop the subscript $\mathcal{L}$ of the order, the supremum, and the infimum. If $\leq$ is a total order then $\mathcal{L}$ is called a chain. A lattice is said bounded if it has a least $\perp$ and a greatest element $T$ (i.e. $\forall a \in \mathcal{L}, \perp \leq a \leq \top$ ). If every non empty family $A \subseteq \mathcal{L}$ has a supremum $\bigvee A$ and an infimum $\bigwedge A$ in $\mathcal{L}$, the lattice $\mathcal{L}$ is said to be a complete lattice. In the following, we consider only complete lattices.

A set $\mathcal{S} \subseteq \mathcal{L}$ such that $\perp \notin \mathcal{S}$ is called a sup-generating family of $\mathcal{L}$ if every element of $\mathcal{L}$ can be written as the supremum of elements of $\mathcal{S}(\forall a \in \mathcal{L}, \exists B \subseteq \mathcal{S}, a=\bigvee B)$. The elements of $\mathcal{S}$ are called sup-generators.

Important examples of lattices are:

- the extended real line $\overline{\mathbb{R}}=\mathbb{R} \bigcup\{-\infty,+\infty\}$, which is a complete chain under the usual order, infimum, and supremum. It is sup-generated by $\mathbb{R}$.

- the set of all subsets of a set $E$ (noted $\mathcal{P}(E)$ ), which is a complete lattice with the partial order defined by the inclusion relation, and the infimum and supremum given by the set intersection and union. It is sup-generated by the singletons of $E$.

- the functions from a set $E$ into a lattice $\mathcal{L}\left(\right.$ noted $\left.\mathcal{L}^{E}\right)$, which is a lattice under the pointwise order $\forall f, g \in$ $\mathcal{L}^{E}, f \leq g \Leftrightarrow \forall x \in E, f(x) \leq_{\mathcal{L}} g(x)$. The infimum and supremum are similarly defined by a pointwise application of the infimum $\Lambda_{\mathcal{L}}$ and supremum $\bigvee_{\mathcal{L}}$ of the underlying lattice. $\mathcal{L}^{E}$ is sup-generated by the pulses: the functions $\delta_{x}^{t}, \forall x \in E, \forall t \in \mathcal{L}, t \neq \perp$ defined by $\forall y \in E, \delta_{x}^{t}(y)=t$ if $x=y, \perp$ otherwise. The properties of $\mathcal{L}^{E}$ depend of the properties of the underlying lattice $\mathcal{L}$. This lattice can be used to represent the images.

\subsection{Set Connection}

Given a set $E$, a connection on $E$ is a family $\mathcal{C} \subseteq \mathcal{P}(E)$ composed of the connected elements of $E$. Formally we say that $\mathcal{C}$ is a connection if [1]: 
1) $\{\emptyset\} \in \mathcal{C}$ : the empty set is connected;

2) $\forall x \in E,\{x\} \in \mathcal{C}$ : the singletons (or points) are connected;

3) $\forall A \subseteq \mathcal{C}, \cap A \neq \emptyset \Rightarrow \bigcup A \in \mathcal{C}$, the union of intersecting connected elements is connected.

\subsection{Hyperconnection}

Being given a finite lattice $\mathcal{L}$ with a sup-generating family $\mathcal{S}$, a h-connection $\mathcal{C}^{+}$on $\mathcal{L}$ is a subset of $\mathcal{L}$ verifying the following conditions [19]:

1) $\perp \in \mathcal{C}^{+}$: the least element is h-connected;

2) $\forall s \in \mathcal{S}, s \in \mathcal{C}^{+}$: the points (i.e. the sup-generators) are h-connected;

3) $\forall A \subseteq \mathcal{C}^{+}, \bowtie A \Rightarrow \bigvee A \in \mathcal{C}^{+}$, the supremum of overlapping h-connected elements is h-connected.

where $\bowtie$ is a predicate on $\mathcal{P}(\mathcal{L})$ called the overlap criterion. This predicate must be decreasing: $\forall A \subseteq \mathcal{P}(\mathcal{L})$, $\bowtie A \Rightarrow \forall b \in \mathcal{L}, \bowtie\{b\} \bigcup A$ meaning that a family of non overlapping elements cannot become overlapping by the addition of new elements. Note that the set connection is simply an h-connection on $\mathcal{P}(E)$ with $\forall A \subseteq \mathcal{C}, \bowtie A \Leftrightarrow \bigcap A \neq \emptyset$.

A simple example of h-connection is made of functions with a unique maximum [19], [20]. This h-connection is defined on the lattice of functions $\mathcal{L}^{E}$ and it is based on a primary set connection $\mathcal{C}_{o}$ on $E$. Then, one says that a function has a unique maximum if it is connected at all levels with respect to the primary set connections. More formally, we define the set of connected functions by: $F=\left\{f \in \mathcal{L}^{E} \mid \forall t \in \mathcal{L}, \bar{f}^{t} \in \mathcal{C}_{o}\right\}$, with $\vec{f}^{t}=\{p \in E \mid t \leq f(p)\}$ the thresholding of $f$ at level $t$. The overlap criterion can be defined as: $\forall\left\{f_{i}\right\} \subseteq$ $\mathcal{L}^{E}, \bowtie\left\{f_{i}\right\} \Leftrightarrow \forall t \in \mathcal{L}, U_{t}=\emptyset$ or $\bigcap U_{t} \neq \emptyset$ with $U_{t}=\left\{{\overrightarrow{f_{i}}}^{t} \mid{\overrightarrow{f_{i}}}^{t} \neq \emptyset\right\}$.

\subsubsection{Hyperconnected operators and openings}

$\mathrm{H}$-connected operators [25] are applications from $\mathcal{L}$ into $\mathcal{P}(\mathcal{L})$ which extract the h-components marked by a supgenerator. Formally, being given a sup-generator $s \in \mathcal{S}$, we define the h-connected operator by:

$$
\begin{aligned}
& \forall a \in \mathcal{L}, \gamma_{s}^{*}(a)= \\
& \left\{h \in \mathcal{C}^{+} \mid s \leq h \leq a, \forall g \in \mathcal{C}^{+}, h \leq g \leq a \Rightarrow g=h\right\}
\end{aligned}
$$

Thus, the h-components are maximal h-connected elements and the h-components of $a \in \mathcal{L}$ are given by:

$$
\gamma^{*}(a)=\bigcup_{s \in \mathcal{S}} \gamma_{s}^{*}(a)
$$

The h-connected opening marked by $s$ is the supremum of the h-components of $a$ above $s$ :

$$
\forall a \in \mathcal{L}, \quad \gamma_{s}(a)=\bigvee \gamma_{s}^{*}(a)
$$

Contrary to connectivity openings in set connection, the result of a h-connected opening is not necessarily connected. Indeed, one can see that $\gamma_{s}(a) \in \mathcal{C}^{+}$if and only if $\# \gamma_{s}^{*}(a)=1$ (where $\# \gamma_{s}^{*}(a)$ is the number of hcomponents in $\gamma_{s}^{*}(a)$ ).

Moreover, the two following properties hold [20]:

1) $a=\bigvee \gamma^{*}(a)$ : an element is the supremum of its h-components;

2) $\forall b, c \in \gamma^{*}(a), b \neq c \Rightarrow b \not c$ : two h-components do not overlap.

\subsubsection{Z-operators}

In [20], the authors propose the interesting notion of $z$ operators. Let $a \in \mathcal{L}$, the equivalence relation $\stackrel{a}{\sim}$ on $\mathcal{S}$ is defined by:

$$
\forall b, c \in \mathcal{S}, \quad b \stackrel{a}{\sim} c \Leftrightarrow \gamma_{b}(a)=\gamma_{c}(a)
$$

Then, the z-operator is defined as the supremum of an equivalence class of $\stackrel{a}{\sim}:$

$$
\forall s \in \mathcal{S}, \quad \zeta_{s}(a)=\bigvee\{b \in \mathcal{S} \mid b \stackrel{a}{\sim} s\}
$$

Finally, the set of all z-zones of $a$ is noted:

$$
\zeta(a)=\left\{\zeta_{s}(a) \mid \forall s \in \mathcal{S}\right\}
$$

The authors of [20] have chosen to base the z-operators on the h-connected openings, but we can also define them in terms of h-connected operators leading to a slightly different definition. Let $\stackrel{a_{*}}{\sim}$ be the equivalence relation defined by:

$$
\forall b, c \in \mathcal{S}, \quad b \stackrel{a_{*}}{\sim} c \Leftrightarrow \gamma_{b}^{*}(a)=\gamma_{c}^{*}(a)
$$

Then, the alternative z-operator* is defined as the supremum of an equivalence class of $\stackrel{a_{*}}{\sim}$ :

$$
\forall s \in \mathcal{S}, \quad \zeta_{s}^{*}(a)=\bigvee\left\{b \in \mathcal{S} \mid b \stackrel{a_{*}^{*}}{\sim} s\right\}
$$

Generally, as h-components can be overlapping, $\zeta_{s}(a) \neq$ $\zeta_{s}^{*}(a)$. For example, consider the set $E=\{a, b, c\}$ and the h-connection $\{\emptyset,\{a\},\{b\},\{c\},\{a b\},\{b c\},\{a c\}\}$ on $\mathcal{P}(E)$ with the overlap criterion defined as false (two elements are never overlapping, this is a valid criterion since it is trivially decreasing). Then, the h-components of $E$ are $\{\{a b\},\{b c\},\{a c\}\}$. Thus, we have $\gamma_{a}(E)=$ $\gamma_{b}(E)=\gamma_{c}(E)=E$ and $\zeta_{a}(E)=\zeta_{b}(E)=\zeta_{c}(E)=E$. But $\gamma_{a}^{*}(E)=\{\{a b\},\{a c\}\}, \gamma_{b}^{*}(E)=\{\{a b\},\{b c\}\}$ and $\gamma_{c}^{*}(E)=\{\{a c\},\{b c\}\}$ thus $\zeta_{a}^{*}(E)=\{a\} \neq \zeta_{a}(E)$. In section 3 , we show that under certain conditions, we have $\forall a \in \mathcal{L}, \forall s \in \mathcal{S}, \zeta_{s}(a)=\zeta_{s}^{*}(a)$. Figure 1 shows an example of function decomposition with the $\mathrm{z}$-operators and the h-connection of function having a unique maximum.

\subsubsection{H-reconstruction}

Being given a marker $m \in \mathcal{L}$ we can define the hyperreconstruction (h-reconstruction) of $a \in \mathcal{L}$ marked by $m$ by [20]:

$$
\lceil a\rceil_{m}=\bigvee_{s \in \mathcal{S}, s \leq m} \gamma_{s}(a)
$$



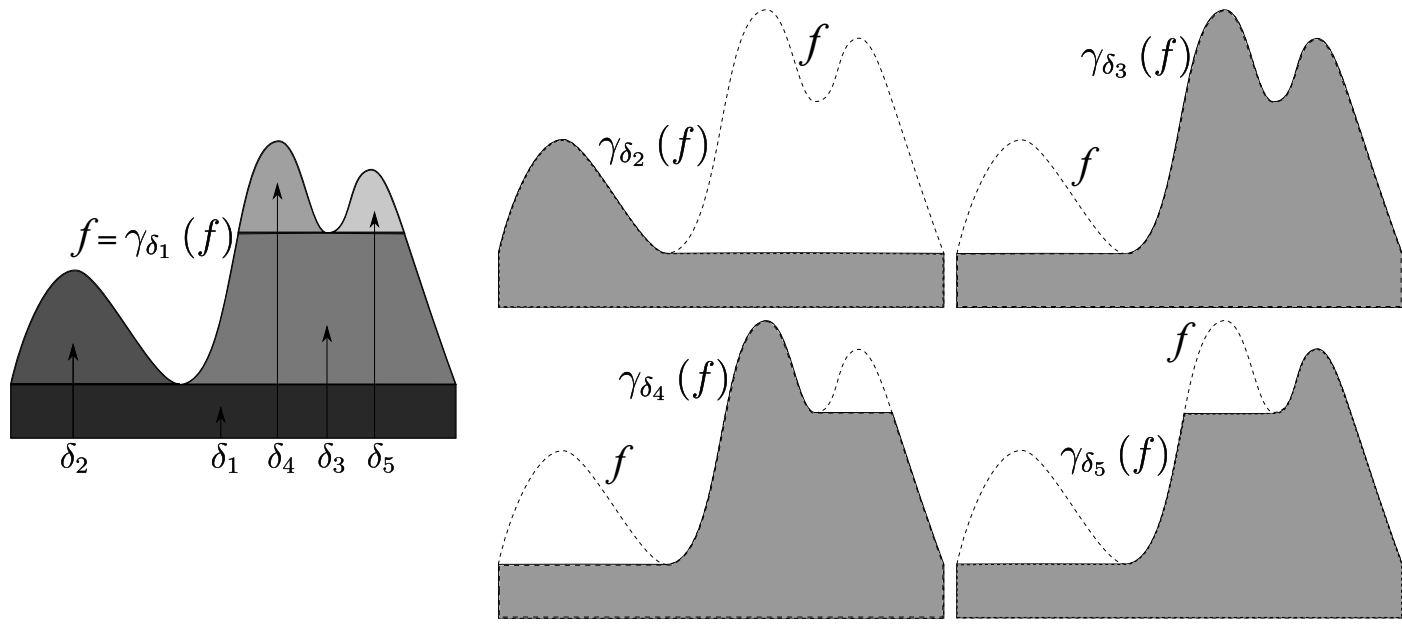

Figure 1. Example of decomposition of function $f$ with the h-connection of functions with a unique maximum: left) function $f$, five pulses $\delta_{1}, \ldots, \delta_{5}$ representatives of the five equivalence classes of the relation $\stackrel{f}{\sim}$. The different gray levels represent the results of the z-operators: $\zeta_{\delta_{i}}(f)$. The sup-generator $\delta_{1}$ (and its equivalence class) are associated to the whole set of h-components of $f$ and thus $\gamma_{\delta_{1}}(f)=f$, right) the h-openings of $\delta_{2}, \ldots, \delta_{5}$.

We propose another definition of the h-reconstruction based on the alternative z-operators:

$$
\lceil a\rceil_{m}^{\zeta}=\bigvee_{s \in \mathcal{S}, s \leq m} \zeta_{s}(a)
$$

The latter is more flexible, the two approaches are compared in Figure 2.

\subsubsection{New properties}

We establish here two new properties that will help us in following proofs. Let $a \in \mathcal{L}$ :

Proposition 1: $\forall\left\{h_{i}\right\} \subseteq \gamma^{*}(a),\left\{h_{i}\right\} \subseteq \gamma^{*}\left(\bigvee\left\{h_{i}\right\}\right)$

Being given a family of h-components, the h-components of its supremum is a superset of the given family. Those two families are generally not equal, take for example the $\mathrm{h}$-connection given in section 2.3.2; the h-components of the element $\{a b c\}$ are $\{\{a b\},\{b c\},\{a c\}\}$. Now, consider the subfamily $\{\{a b\},\{b c\}\}$, the supremum of this family is equal to $\{a b c\}$ and the h-components of this supremum is a strict superset of the family. This property is important for the theory as it shows that the deletion of an h-component of an image may leave the image unchanged. The corollary of this observation is that deleted $\mathrm{h}$-components of an image may reappear in the result.

Proposition 2: $\forall s \in \mathcal{S}, \forall h^{\prime} \in \gamma^{*}(a)$, if $s \leq h^{\prime}$ and $\gamma_{s}(a)=h \in \mathcal{C}^{+}$then $h=h^{\prime}$.

If the h-opening of $a$ marked by the sup-generator $s$ is a h-component then there is no other h-component of $a$ above $s$.

After this presentation of the current h-connection theory, in the next section, we propose a new axiomatic of h-connections. Indeed, the weaknesses of the current axiomatic are discussed, and a novel solution is then proposed. In the following, we assume the existence of a complete lattice $\mathcal{L}$ with the sup-generating family
$\mathcal{S}$ and the h-connection $\mathcal{C}^{+}$. Moreover, we assume that the number of h-components of each element of $\mathcal{L}$ is finite. One can note that this condition is satisfied when working on discrete images of a finite domain.

\section{TOWARD A NEW AXIOMATIC}

Despite the successful developments of h-connections in recent works [20], [22], [23], [24], [25], [26], the theory of h-connections is still not satisfactory. All of the successes obtained have been based on specific h-connections, whereas only a few general properties have been established. This lack of theoretical results comes from the very broad definition of h-connections. The third axiom (the overlap criterion) is especially problematic. On one hand, this axiom is satisfactory because it formalizes the intuitive union based approach. On the other hand, the definition of the overlap criterion is so wide that, in practice, this third axiom does not bring any meaningful property to the h-connection (for example, consider the overlap criterion which is always false, and the third axiom is never applicable). Instead of a constantly false overlap criterion, one can also define the following less trivial valid overlap criterion: $\forall \mathcal{C}^{+} \in \mathcal{L}, \forall A \subseteq \mathcal{C}^{+}, \bowtie$ $A \Leftrightarrow\left(\forall B \subseteq A, \bigvee B \in \mathcal{C}^{+}\right)$.

To harden the definition of h-connections, we propose the fundamental property that an h-connection must provide for each element a decomposition in hcomponents that is necessary and sufficient. With the current theory it is clear that the decomposition in hcomponents is sufficient in the sense that an element is equal to the supremum of its h-components. But in general this decomposition is not necessary in the sense that only a subset of the h-components of an element can be necessary to retrieve the element by supremum.

More formally, one can define the following properties $\forall a \in \mathcal{L}:$ 


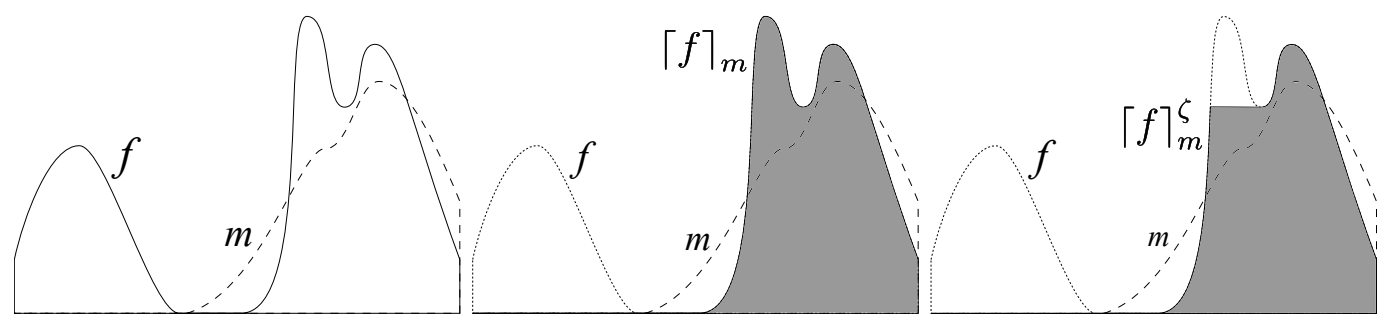

Figure 2. The h-reconstruction operator with the h-connection of functions with a unique maximum. From left to right: function $f$ and marker $m$, h-reconstruction $\lceil f\rceil_{m}$ and alternative h-reconstruction $\lceil f\rceil_{m}^{\zeta}$.

\section{P-1 - $\forall h^{\prime} \in \gamma^{*}(a), \bigvee\left\{h \in \gamma^{*}(a) \mid h \neq h^{\prime}\right\}<\bigvee \gamma^{*}(a):$ all h-components of an element are necessary to describe the element. \\ P-2 - $\forall\left\{h_{i}\right\} \subseteq \gamma^{*}(a), \forall h \in \gamma^{*}(a), h \leq \bigvee\left\{h_{i}\right\} \Rightarrow$ $h \in\left\{h_{i}\right\}$ : an h-component cannot be covered by other h-components. \\ P-3 - $\forall h \in \gamma^{*}(a), \exists s \in \mathcal{S}, \gamma_{s}^{*}(a)=h$ : each h- component of an element can be individually retrieved through an h-opening.}

Then, the following proposition holds:

Proposition 3: Properties P-1, P-2, and P-3 are equivalent. In the following, we will say that a connection is accessible if the previous properties hold.

P-1 and P-2 are two direct formulations of the "necessity" condition, the first one from a global point of view, the second from a local point of view. P-3 shows that this condition naturally comes to the notion of special groups of sup-generators that give access to a unique h-component through an h-opening. From the image processing point of view, this means that every $\mathrm{h}$-component of an image can be selected individually with a simple opening. Accessible h-connections also have harder properties:

Proposition 4: If $\mathcal{C}^{+}$is accessible, being given a family of h-components, the h-components of its supremum is the same family: $\forall\left\{h_{i}\right\} \subseteq \gamma^{*}(a),\left\{h_{i}\right\}=\gamma^{*}\left(\bigvee\left\{h_{i}\right\}\right)$

Compared to proposition 1, this version is harder and it ensures that by selecting a family of h-components (corresponding to a given criterion), its reconstruction by the supremum operator will not introduce new hcomponents. From the image processing point of view, this ensures that, when performing an h-connected filtering, a deleted h-component cannot re-appear after the reconstruction of the selected h-components. Another interesting property of accessible h-connections concerns z-operators:

Proposition 5: If $\mathcal{C}^{+}$is accessible, the equivalence relations $\stackrel{a}{\sim}$ and $\stackrel{a_{*}}{\sim}$ are equivalent: $\forall s_{1}, s_{2} \in \mathcal{S},\left(s_{1} \stackrel{a}{\sim} s_{2}\right) \Leftrightarrow$ $\left(s_{1} \stackrel{a_{*}}{\sim} s_{2}\right)$.

This property removes the necessity of operating a choice between the two distinct approaches when dealing with z-operators. Thus it simplifies the process of creating image filters based on z-zones.

\section{HYPERCOMPONENT TREE}

In this section, we show how decompositions using accessible (according to proposition 3) h-connections can lead to a hierarchical representation in the lattice of functions (images). By definition, the h-components of an image are not ordered and thus the usual component tree representation is not suitable. In [20], [31], the authors propose a definition of a grayscale component tree for a particular h-connection. Here, we propose a more general definition based on z-zones (note that as $\mathcal{C}^{+}$ is accessible, then all z-zone definitions are equivalent). The global strategy is then to furnish the z-zones with a partial order relation noted $\preceq$ and to prove that the Hasse diagram of the z-zones of an element is a tree.

As the tree is a representation of the decomposition of the image into its h-components, its properties will depend of the chosen h-connection.

\subsection{Partial Order of the Z-Zones}

Let $\mathcal{I}=\mathcal{L}^{E}$ be the lattice of images with $\mathcal{L}$ a lattice, $E$ a domain. The support of a function $f \in \mathcal{I}$ is the set of points where the function is different from $\perp$ : $\operatorname{supp}(f)=$ $\{p \in E \mid f(p) \neq \perp\}$. We first define a binary relation $\preceq$ on $\mathcal{I}$ :

$$
\begin{aligned}
\forall x, y \in \mathcal{I}, \quad x \preceq y & \Leftrightarrow \\
(\operatorname{supp}(x) & \subseteq \operatorname{supp}(y)) \wedge(y / \operatorname{supp}(x) \leq x)
\end{aligned}
$$

where $y / \operatorname{supp}(x)$ is the function $y$ restricted on $\operatorname{supp}(x)$ :

$$
\forall p \in E, \quad y_{/ \operatorname{supp}(x)}(p)= \begin{cases}y(p) & \text { if } p \in \operatorname{supp}(x) \\ \perp & \text { otherwise }\end{cases}
$$

Proposition 6: $\preceq$ is a partial order on $\mathcal{I}$.

With this special order, a function $x$ is lower than another function $y$ if the support of $x$ is included in the support of $y$ and if the value of $x$ is larger than that of $y$ on the support of $x$ (see Figure 3). Moreover, the pair $(\mathcal{I}, \preceq)$ is a lattice with the infimum $\wedge \preceq$ and supremum $\vee_{\preceq}$, for all $x, y \in \mathcal{I}$ defined as:

$$
\begin{aligned}
\forall p \in E, \quad(x \wedge \preceq y)(p) \\
\quad= \begin{cases}x(p) \vee_{\mathcal{L}} y(p) & \text { if } p \in \operatorname{supp}(x) \cap \operatorname{supp}(y) \\
\perp & \text { otherwise }\end{cases}
\end{aligned}
$$




$$
\begin{aligned}
& \forall p \in E,\left(x \vee_{\preceq} y\right)(p) \\
& = \begin{cases}x(p) \wedge_{\mathcal{L}} y(p) & \text { if } p \in \operatorname{supp}(x) \cap \operatorname{supp}(y) \\
x(p) & \text { if } p \in \operatorname{supp}(x) \text { and } p \notin \operatorname{supp}(y) \\
y(p) & \text { if } p \in \operatorname{supp}(y) \text { and } p \notin \operatorname{supp}(x) \\
\perp & \text { otherwise }\end{cases}
\end{aligned}
$$

Figure 3 demonstrates the basic properties of $\preceq$ and its associated infimum and supremum operators.

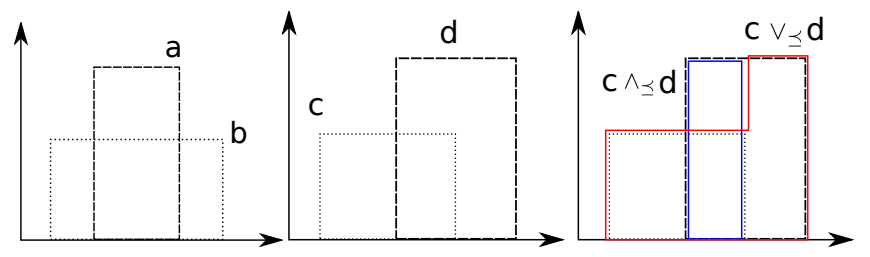

Figure 3. Left image: two functions $a$ and $b$ such that $a \preceq b$. Middle image: two functions $c$ and $d$ that are not comparable according to $\preceq$. Right image: the infimum $c \wedge \preceq d$ (blue) and supremum $c \vee_{\preceq} d$ (red) of the functions $c$ and $d$ of the middle image.

\subsection{Tree Representation Definition}

Then, a graph based representation of an image $a \in \mathcal{I}$ is obtained using the Hasse diagram $\left(G_{a}, V_{a}\right)$ of the z-zones defined by the order $\preceq$. The graph $\left(G_{a}, V_{a}\right)$ is defined by:

$$
\begin{gathered}
G_{a}=\zeta(a) \cup\{\perp\} \\
V_{a}=\left\{(x, y) \in G_{a}^{2} \mid x \neq y, x \preceq y,\right. \\
\left.\left(\forall z \in G_{a}, x \preceq z \prec y \Rightarrow x=z\right)\right\}
\end{gathered}
$$

where $\prec$ means strictly lower than: $z \prec y \Leftrightarrow(z \preceq y) \wedge$ $(z \neq y)$. Property P-3 guarantees that a mapping exists between groups of sup-generators (z-zones) and hcomponents, thus each h-component has a dedicated node in the graph. We will now give a sufficient (but not necessary) condition to ensure that the Hasse diagram is an acyclic graph so that it can be considered as a tree rooted by $\perp$. The least element $\perp$ is added to ensure the tree structure (otherwise it might be a forest), in consequence we will omit it when it is not necessary.

Proposition 7: If the elements of $G_{a}$ are either disjoint or comparable (i.e. $\forall x, y \in G_{a}, x \wedge \preceq y \neq \perp \Rightarrow(x \preceq y) \vee$ $(y \preceq x))$, then the graph $\left(G_{a}, V_{a}\right)$ is acyclic.

Thus, the only requirement to ensure that the z-zones of an image in $\mathcal{L}^{E}$ can be represented as a tree structure with respect to the partial order $\preceq$ is that $\mathcal{C}^{+}$fulfills the condition of proposition 7. Figure 4 shows an example of an h-component tree with the h-connection of functions with a unique maximum. With this particular $\mathrm{h}$-connection the result is equivalent to the grayscale component tree proposed in [20], [31].

The h-component tree can then be used in the same framework as the traditional Max-Tree [27]: equip each node of the tree with appropriate attributes, perform tree pruning based on the node attributes, and if necessary,

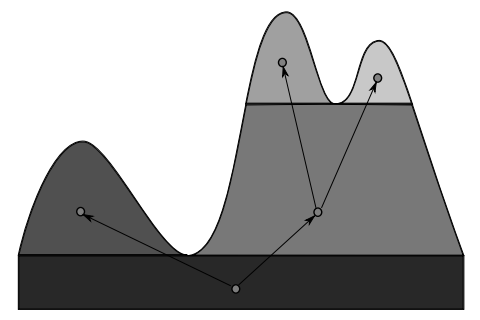

Figure 4. The h-component tree obtained with the hconnection of functions with a unique maximum ( $\perp$ is not represented as it is not needed to root the graph).

reconstruct an image from the reduced tree using the reconstruction operator $\lceil a\rceil_{m}^{\zeta}$.

In the next section, we demonstrate the capabilities of the h-component tree with a more complex and interesting h-connection.

\section{Application}

We propose applying the theoretical approach described above to a fuzzy h-connection recently proposed in [22]. In this article, the authors have proposed an extension of the fuzzy connectivity measure [32] and have shown that it can be expressed in the theory of h-connections.

In this section, we apply our method to this fuzzy h-connection and demonstrate its capabilities on simple filtering operations. We also extend the definition of the h-connection to handle multiband images.

\subsection{Fuzzy H-Connection}

We now consider the lattice $\mathcal{I}$ of functions from a discrete domain $E$ to the interval $[0,1]$ (which is a complete chain). We assume that $E$ is equipped with a primary set connection $\mathcal{C}$. We define the connectivity measure $c_{f}$ of the image $f$, for all $x, y \in E$ (definition adapted from [22]):

$$
c_{f}(x, y)=\max _{M \in P_{x, y}} \min _{p \in M} f(p)
$$

with $P_{x, y}=\{M \in \mathcal{C} \mid x \in M, y \in M\}$ the set of all connected sets containing $x$ and $y$. This can be equivalently defined in terms of graph connectivity by the set of all paths from $x$ to $y$. If $P_{x, y}$ is empty, we set $c_{f}(x, y)=0$ meaning that $x$ and $y$ are not connected. The fuzzy hconnection $\mathcal{C}_{f, \tau}^{+}$of level $\tau \in[0,1]$ is then defined by (definition adapted from [22]):

$$
\begin{aligned}
& \mathcal{C}_{f, \tau}^{+}=\{f \in \mathcal{I} \mid \\
& \left.\quad \forall x, y \in E, 1-\min (f(x), f(y))+c_{f}(x, y) \geq \tau\right\}
\end{aligned}
$$

As stated in section 3, the overlap criterion is not given. The discriminant points of the function used to determine the connectivity measure are indeed regional maxima ( $M \in E$ is said to be a regional maxima of $f$ if it exists a level $t \in[0,1]$ such that $M \subseteq \vec{f}^{t}, M \in \mathcal{C}$ and $\forall p \in M, f(p)=t$ ). This property is illustrated in Figure 5. 


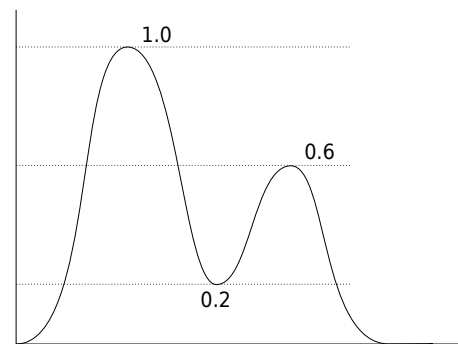

Figure 5. This function has two regional maxima at levels 1.0 and 0.6 . The connectivity measure $c_{f}$ between the maxima is equal to 0.2 . Thus, this function belongs to $\mathcal{C}_{f, \tau}^{+}$ for all $\tau \leq 0.6$.

The set $\mathcal{C}_{f, \tau}^{+}$is a h-connection as it contains the least element and the pulses. It is clearly accessible trough the principal mode of each h-component (P-3) and $[0,1]$ is a chain. Moreover, proposition 17 of [22] establishes that fuzzy h-components of an image are isomorphic to subtrees of the Max-Tree [5], [13] and the authors provide a method to easily extract each fuzzy h-component individually from the Max-Tree representation. In addition, their method shows that $\mathcal{C}_{f, \tau}^{+}$fulfills proposition 7 and thus, $\mathcal{C}_{f, \tau}^{+}$can be used to build a h-component tree.

One can notice that if the primary set connection $\mathcal{C}$ is invariant to translations, rotations, and homothetic transformations (like the four- or eight-connected neighborhood), then the fuzzy h-connection built over this set connection is also invariant to these transformations, as is the fuzzy h-component tree. Nevertheless, the fuzzy h-connection is only invariant to gray level value translations of the image apart for the special case of $\tau=0$ : it is then invariant to any increasing gray level transformation.

Then, the method to compute an h-component can be adapted to build the complete h-component tree. The proposed procedure is composed of both algorithms 1 and 2. The pseudocode assumes that each node is equipped with two attributes, the level and the peak level (highest level in its branch), and a function child(n) that returns the $\mathrm{n}$-th child. The first algorithm increases the level of each node and deletes useless branches (branches which do not have sufficient contrast to generate an $\mathrm{h}$-component). The procedure delete node, deletes the given node and gives all its children to its father, while the procedure delete branch deletes the given node and all its children. At the end of the algorithm, all attributes can be correctly computed according to the $\mathcal{C}_{f, \tau}^{+}$h-connection. The second algorithm aims at restoring the good point list to all nodes according to their new levels. It uses a stack structure (with traditional push and pop operators) to explore the branch up to the needed level. While the Max-Tree computation over an image of 1 million pixels in double precision takes 14 seconds with Najman and Couprie's algorithm [13] for 481384 nodes, the transformation into the h-component tree takes 5 seconds for 3113 nodes in the result (code
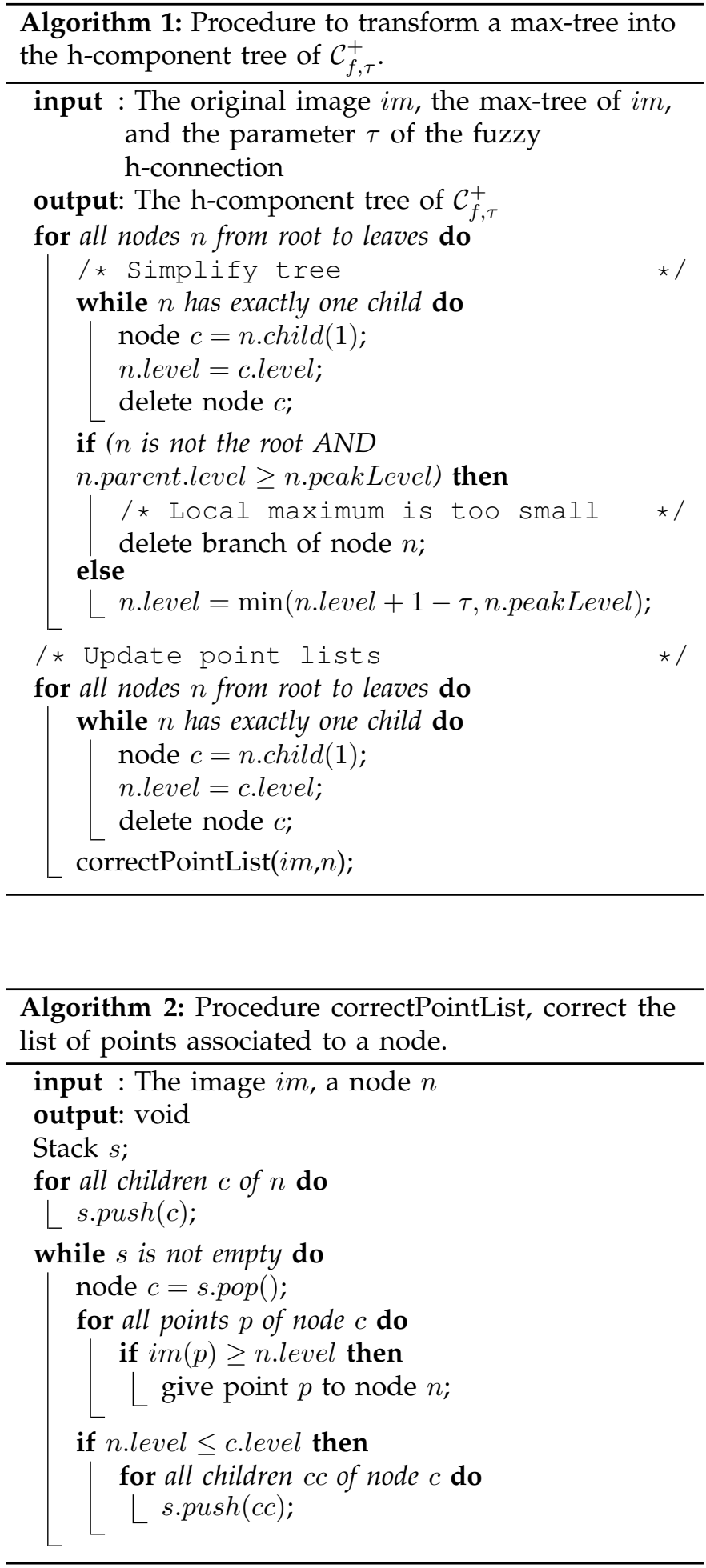

Moreover, according to these algorithms, it is clear that any pruning of the h-component tree of the fuzzy hconnection performed according to an increasing criterion [27] followed by an h-reconstruction will produce an idempotent, increasing, and anti-extensive morphological operator: i.e. a morphological opening. 

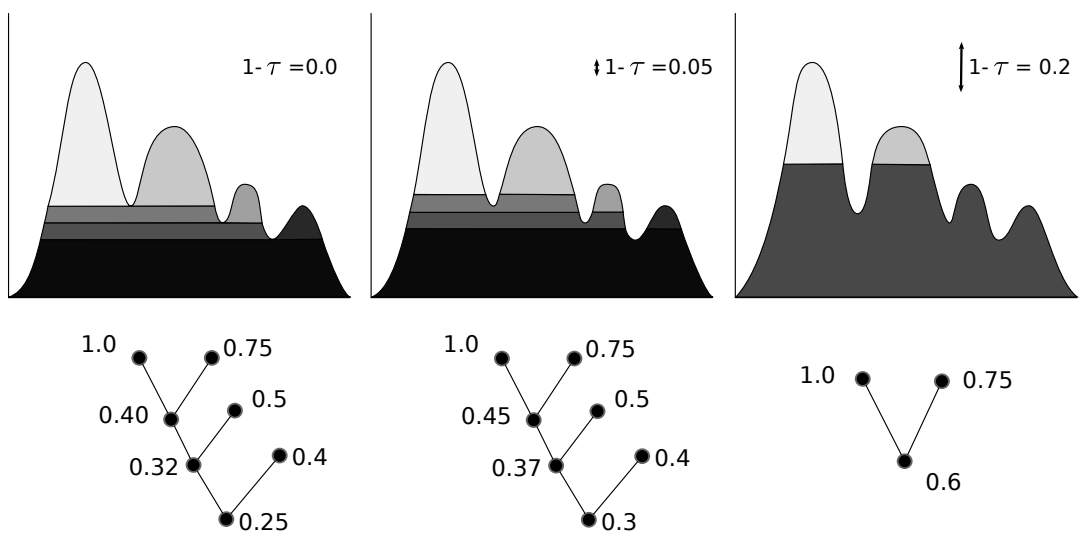

Figure 6. The h-component tree for different values of $\tau$. For each value of $\tau$, the first row shows a 1D representation of the signal with the associated z-zones. The second row represents the h-component tree with the level of each node. The left case is equivalent to the Max-Tree where all nodes having a unique child have been removed.

\subsection{Grayscale Images}

We have first tested the gain of our approach in terms of tree complexity. Figure 6 shows the evolution of the h-component tree in function of $\tau$. When $\tau=1$, the tree is simply the Max-Tree where all nodes having a unique child have been deleted; one can note that in real images, this generally does not modify the tree a lot, as even a small noise generates a lot of leaves in the MaxTree. It is also equivalent to the h-component tree of the $\mathrm{h}$-connection of functions having a unique maximum. When $\tau$ starts increasing in the second image, the level of the nodes raises. In the third image, $\tau$ is large enough to absorb two of the four local maxima in a unique node.

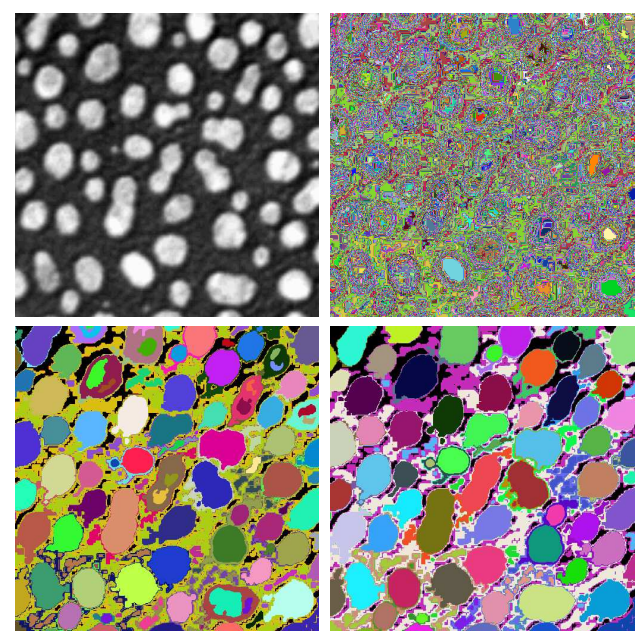

Figure 7. (From left to right, top to bottom): input image and segmentation results with different values of $\tau: 1.0$, 0.9 , and 0.8 .

Figure 7 illustrates the effect of $\tau$ on a simple image. The first figure is the grayscale image. The primary set connection $\mathcal{C}$ used in this example is the four neighborhood. To produce the other three images, each node of the h-component tree has been labeled with a random

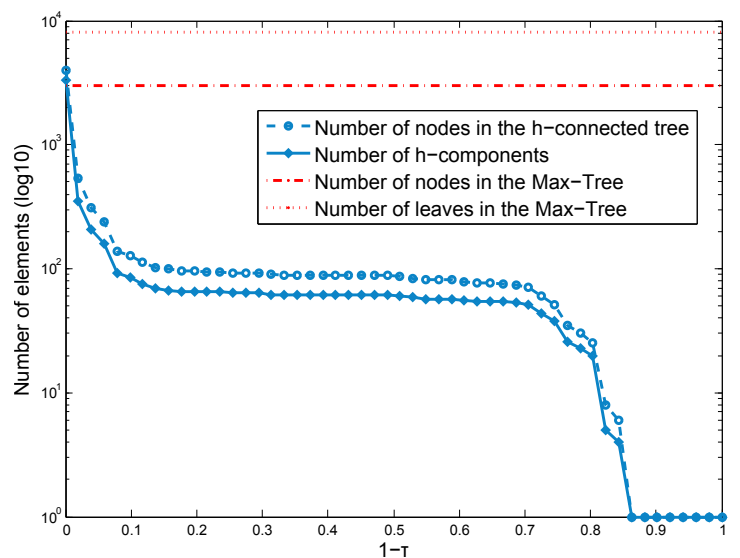

Figure 8. Number of nodes and h-components in function of $\tau$ compared to the number of nodes and leaves in the Max-Tree (image in Figure 7). Y-axis is in log scale.

color. Then, the pixel values are given by the label of the highest node they belong to. When $\tau=1.0$, there are 4019 nodes in the tree for $3290 \mathrm{~h}$-components. The number of nodes drops to 127 (respectively 97) and the number of h-components to 85 (respectively 66) when $\tau$ equals 0.9 (respectively 0.8). We can observe that for $\tau=0.8$, all blobs are uniquely identified by an hcomponent (a leaf in the h-component tree). They are bigger than they appear in the original image as the z-zones extend as much as possible according to the connection measure.

The number of nodes and h-components in the hcomponent tree in function to $\tau$ compared to the number of nodes and leaves in the Max-Tree is detailed in Figure 8. We can see that the number of nodes decreases quickly when $\tau$ increases; it then reaches a large stable period to finally fall to one. This large stable period can be associated to the optimal value of $\tau$ and suggests that this parameter can be roughly defined and that it is robust over a large interval.

In Figure 9, we propose a comparison with other 

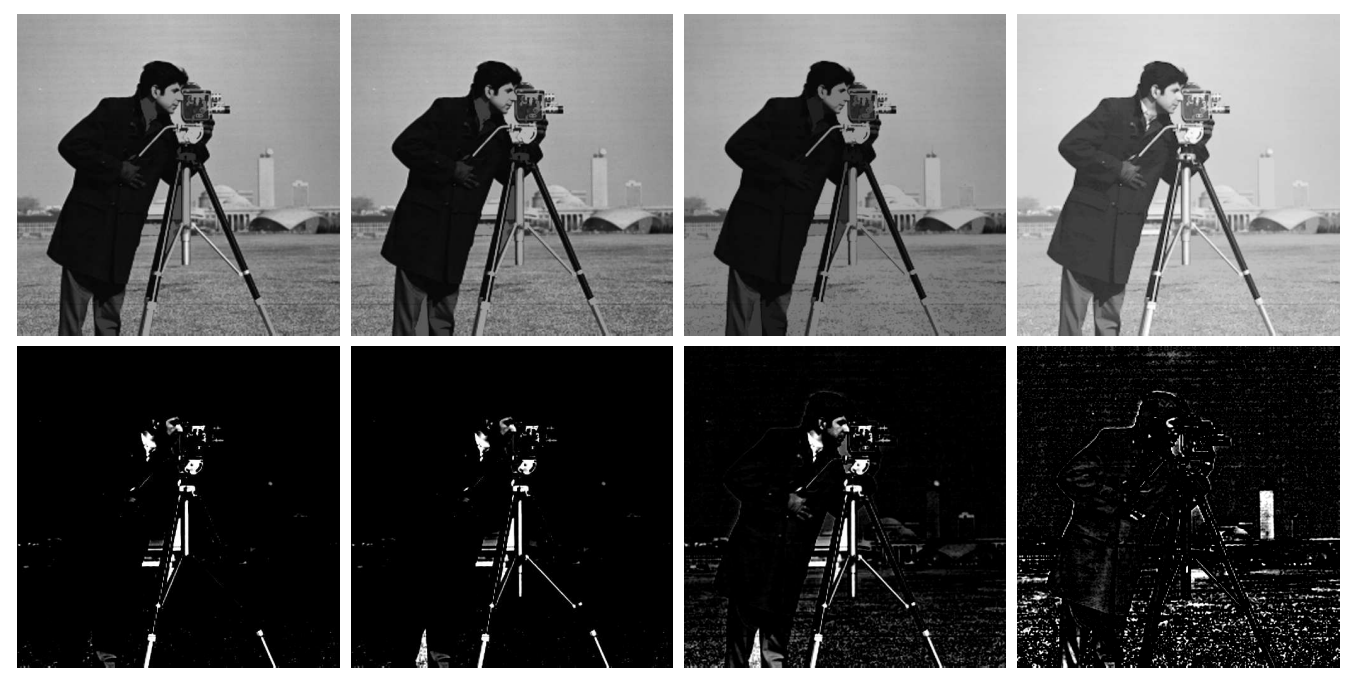

Figure 9. Connected filtering of the traditional camera image. The first row shows the results of the filters and the second row shows the difference with the original image (contrast increased). From left to right: area filter (1000 pixels) based on the h-component tree with the fuzzy h-connection $\mathcal{C}_{f, 0.8}^{+}$, area filter (1000 pixels) using the kzone reconstruction method [23], [24] ( $\mathrm{k}=0.2$ ), area filter (1000 pixels) using traditional Max-Tree [5] and dynamic opening proposed in [33]. The results show that both the h-component tree and the k-zone approach lead to a better preservation of textures. The proposed approach concentrates only on distinct zones with sufficient contrast, while internal details of objects, textures, and softly connected objects are preserved.

connected filters on the traditional camera image. The test implies three area connected filters which consists in deleting all nodes having an area outside a given range: with the proposed fuzzy h-component tree, with the k-zone method [23], [24], with the traditional MaxTree [5], and finally the dynamic opening [27], [33]. The results show that the h-component tree and the k-zone approach lead to a better preservation of textures. Only clearly separated zones are removed by these filters, softly connected areas like the buildings in the background are considered as connected objects as well as the texture of the grass. The edges of the cameraman are also not affected by these filters. On the other hand, the space between the tripod is falsely considered by all area filters as disconnected to the background and is removed. This is due to the high contrast between the legs of the tripod and the background that cannot be absorbed neither by the $\tau$ parameter nor the k-zones. This difficulty could be overpassed using a primary set connection $\mathcal{C}$ of second generation [18] instead of a simple four-connected neighborhood.

\subsection{Multiband Images}

In this section, we present how the fuzzy h-connection can be adapted to handle multiband images. We now consider functions from the domain $E$ into $[0,1]^{n}$ with $n \geq 1$ the number of bands. The parameter $\tau$ becomes a vector in $[0,1]^{n}$ and we must furnish the value space with a total vectorial order. Theoretically, any total vectorial order [34] can be used to furnish $[0,1]^{n}$ with a lattice structure. However, proposed algorithm 1 relies on additive operations and thus requires that the vectorial ordering is translation invariant: $\forall x, y, z \in[0,1]^{n}, x \leq$ $y \Leftrightarrow x+z \leq y+z$.

Figure 10 shows an example of multiband connected filtering on astronomical data. The original image is a five band image of the galaxy PGC35538 taken from the Sloan Digitalized Sky Survey (http://www.sdss.org/). This example shows the ability of the h-component tree and the fuzzy h-connection to selectively remove small regions while fully preserving the rest of the image.

The image values were first mapped to $[0,1]^{5}$ using a sigmoid transform. This transform has the advantage of reducing the importance of the background noise and very bright features, while features of medium brightness are emphasized. The parameter $\tau$ can be automatically set proportional to the standard deviation of noise (note that the standard deviation of noise is easily obtained in astronomical images by measuring it on the background). The chosen vectorial order is a lexicographic order which is the simplest ordering that fulfills the translation invariance property. Finally, the bands where ordered by decreasing values of signal to noise ratio. Using $\tau$ equal to three times the standard deviation of noise, the number of nodes and leaves in the h-component tree compared to the number in the Max-Tree reduces from 65085 nodes to 496 nodes and from 6430 leaves to 249 leaves.

The test consists in performing an area filter (area greater than 30 pixels) using the proposed h-component tree compared to a traditional vectorial area filter with a Max-Tree [35]. The tests using the Max-Tree were performed twice, the first time using the same area limit as with the h-component tree ( 30 pixels) and a second time using a smaller area limit (10 pixels) as nodes in the 

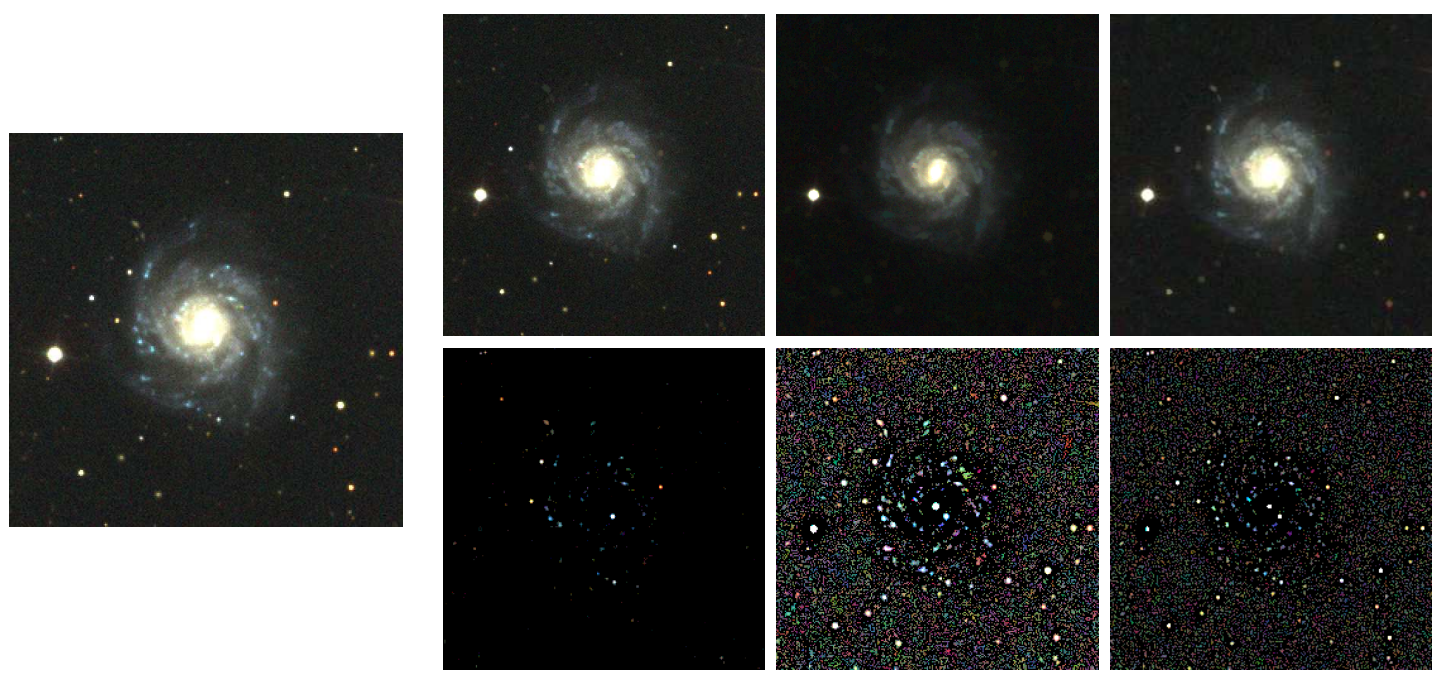

Figure 10. Removal of small features from galaxy image with area filtering. The left image is an observation of galaxy PGC35538 in five bands from near ultra violet to near infrared (only three band are used in the color composition). Then, the first row shows the result of the area filter using from left to right: the h-component tree (area greater than 30 pixels), the Max-Tree (area greater than 30 pixels) , the Max-Tree (area greater than 10 pixels). The second row shows the difference between the first row and the original image. All images are displayed with an enhanced contrast. The results show that the proposed approach is suitable for multiband image processing. It provides a better preservation of the galaxy morphological features (shape of the arms, the very bright central zone is not cut) and the background is not affected.

h-component tree tend to be larger than in the Max-Tree.

The experiment shows that our approach is very accurate accurate in extracting small features which have sufficient contrast from a larger object. The background is hardly affected, and more importantly, the morphology of the galaxy is not affected (the very bright central zone is not cut and morphological details in the arms are preserved). On the contrary, the Max-Tree modifies the background and the fine structures of the galaxy.

\subsection{Document Image Binarization}

The last experiment concentrates on the issue of document image binarization. Binarization is an important step in document analysis and it requires robust methods able to manage degraded document images of different natures (handwritten, printed) with various scales and varying contrasts. We propose a novel method which is based on background removal using the h-component tree. The method processes the image in three steps:

1) the image values are mapped to $[0,1]$ with a linear transform and the background is extracted using the h-component tree;

2) an adaptive threshold is applied;

3) a post-processing is applied to remove small holes and artifacts.

The background identification is based on the evolution of the area of the h-component tree nodes compared to their gray level. The main idea is that, in the foreground, the evolution of the curve level-area respects two criteria: a global one on the slope of the curve and a local one on its derivative. Formally, being given a leaf $L$, we look for the largest set of nodes $\left\{N_{0}, \ldots, N_{m}\right\}$ of respective level $T_{i}$ and area $A_{i}$, such that $L=N_{0}, \forall i=1, \ldots, m, N_{i}$ is the father of $N_{i-1}$ and:

$$
\begin{gathered}
\forall i=1, \ldots, m: A_{i}<c_{3} \vee \frac{A_{i}-A_{0}}{T_{i}-T_{0}}<c_{1} \\
\forall i=1, \ldots, m: A_{i}<c_{3} \vee \frac{A_{i}-A_{i-1}}{T_{i}-T_{i-1}}<c_{2}
\end{gathered}
$$

with three thresholds, $c_{1}, c_{2}$ and $c_{3}$. $c_{1}$ constrains the global slope and $c_{2}$ constrains its derivative. $c_{3}$ allows one to neglect small nodes for which the noise on the level-area curve may be too strong. Finally, $N_{m}$ is considered as the first node above the background level in the branch of $L$. The last constraint is an absolute contrast criterion: if the difference between the level of $L$ and the level $N_{m}$ is smaller than $c_{4}$, the whole branch is discarded. Otherwise, the local background is obtained by the reconstruction of the tree where the branch of $N_{m}$ has been removed. The global background is defined as the infimum of all local backgrounds.

Thresholds $c_{1}$ and $c_{2}$ can handle large variations in scale assuming that the contrast of the foreground is sufficient. $c_{3}$ and $c_{4}$ are absolute values referring to notions of relevant contrast and size. In the following, we use the values: $c_{1}=-8 \times 10^{4}, c_{2}=-2 \times 10^{6}, c_{3}=2 \times 10^{3}$, $c_{4}=0.1$ and the parameter $\tau$ of the fuzzy connection is set to $10 / 255$.

Figure 11 shows three results of background filtering on handwritten and printed document images extracted from the DIBCO 2009 dataset [28]. The first handwritten document image suffers from a severe defect due to coffee stains. The second handwritten document image 
is affected by large variations in its background level and in text contrast. Finally, the last computer printed document image is characterized by large variations in scale. We can observe that in the three cases the background is very well flattened.

The binarization step relies on an adaptive thresholding strategy inspired by the method 26 (S. Lu and C.L. Tan from the Institute for Infocomm Research, Singapore) of the DIBCO 2009 contest [28]. First the contrast is improved with an histogram cut (saturation of the $2 \%$ brightest pixels), and the edges of the image are detected using a simple Sobel operator together with an Otsu thresholding. Then, the local threshold value relies on the values of edge pixels in a window of $32 \times 32$ pixels: if there is less than 10 edge pixels in the window, their mean value is used, otherwise the threshold is set to the first quartile of the edge values. Finally, the result is enhanced using a binary closing by reconstruction and a binary opening by reconstruction with a circular structuring element with a 5-pixel diameter. Figure 12 shows the result of the binarization process on two images of the DIBCO benchmarking dataset [28].

Finally, we measured the performances of the method over the whole DIBCO benchmarking dataset. The dataset is composed of 5 handwritten images and 5 printed images especially designed to include all major difficulties of document binarization. The ground-truth of the dataset is composed of manual segmentations of the images given by the authors of the dataset. We transformed color images into grayscale images by computing the mean of the RGB channels. The performances are evaluated in terms of F-Measure and Peak Signal To Noise Ratio (PSNR) which are the two highlighted measures (among four) involved in the DIBCO 2009 contest:

$$
\begin{array}{r}
\text { F-Measure }=\frac{2 \times \text { Precision } \times \text { Recall }}{\text { Precision }+ \text { Recall }} \\
\text { Precision }=\frac{\mathrm{TP}}{\mathrm{TP}+\mathrm{FN}}, \quad \text { Recall }=\frac{\mathrm{TP}}{\mathrm{TP}+\mathrm{FP}} \\
\mathrm{PSNR}=10 \log _{10}\left(\frac{\mathrm{TN}+\mathrm{TP}+\mathrm{FN}+\mathrm{FP}}{\mathrm{FN}+\mathrm{FP}}\right)
\end{array}
$$

with TP, TN, FP, and FN being respectively the number of true positives, true negatives, false positives, and false negatives. The PSNR formula assumes that the difference between black and white is equal to 1 . Both measures have to be maximized. The results are summarized in Table 1. The proposed approach gives the best result with $\tau=10 / 255$ : it is first placed according to the F-Measure and second placed according to the PSNR (there were 43 participants at the contest). Moreover, one can note that the the binarization step could be greatly improved using a more accurate and robust edge detector but our aim here was to show how document analysis can benefit from h-component tree image representation.

The method has also been submitted to the Handwritten Document Image Binarization Competition 2010 [36] which took place at the 12th International Conference on Frontiers in Handwriting Recognition and it placed 4th among 16 participants [36]. The method was applied using the best set of parameters found for the DIBCO 2009 dataset, which shows the robustness of the parameters.

\begin{tabular}{|l|c|c|}
\hline Method & F-Measure & PSNR \\
\hline \hline $\begin{array}{l}\text { Winner of the contest: S. Lu and C.L. Tan } \\
\text { from the Institute for Infocomm Research, }\end{array}$ & 91.24 & 18.66 \\
$\begin{array}{l}\text { Singapore } \\
\text { Second-place winner of the contest: J. Fab- } \\
\text { rizio and B. Marcotegui from UPMC and }\end{array}$ & 90.06 & 18.23 \\
$\begin{array}{l}\text { CMM, France } \\
\text { Third-place winner of the contest: D. Rivest- }\end{array}$ & 89.34 & 17.79 \\
$\begin{array}{l}\text { Hénault, R.F. Moghaddam and M. Cheriet } \\
\text { from University of Quebec, Canada }\end{array}$ & & \\
Proposed approach $(\tau=0)$ & 91.25 & 18.23 \\
Proposed approach $(\tau=10 / 255)$ & 91.26 & 18.30 \\
Proposed approach $(\tau=20 / 255)$ & 87.85 & 17.11 \\
\hline
\end{tabular}

Table 1

Results on the DIBCO benchmarking dataset. Our

method $(\tau=10 / 255)$ is placed first according to the

F-Measure and second according to the PSNR.

\section{Conclusion and Perspectives}

We have proposed an evolution of the notion of hconnection motivated by the desirable property that the decomposition of an element in connected components should provide a model that is necessary and sufficient to describe this element. Due to this evolution, we have set out several new important properties for image processing which insure the consistency of h-connected filters. In this formulation, the h-connection no longer needs to be furnished with an overlap criterion. Nevertheless, even if the overlap criterion is not a key point of the h-connection theory, it may remain a valuable notion to formalize the notion of intersection of h-elements.

We have also designed a general method to obtain a hierarchical representation of the decomposition in hcomponents which has proven to be efficient and intuitive for the creation of attribute filters or for detection tasks. This representation is based on the notion of $\mathrm{z}$ zones defined by an equivalence relation on the supgenerating family of the lattice, all points of the z-zone being assigned to the same group of h-components.

These theoretical developments were illustrated using a fuzzy h-connection recently proposed in [22]. We proposed an algorithm to transform a Max-Tree into an hcomponent tree based on this fuzzy h-connection. Then, we demonstrated the behavior of this tree in terms of complexity and filtering capacity. We showed that the method was easily applicable to multiband images with an example in multiband astronomical imaging. Finally, we compared a novel h-component tree based binarization method with the DIBCO 2009 benchmarking dataset [28]: the very good results assert the interest of this tool for various image processing issues. 

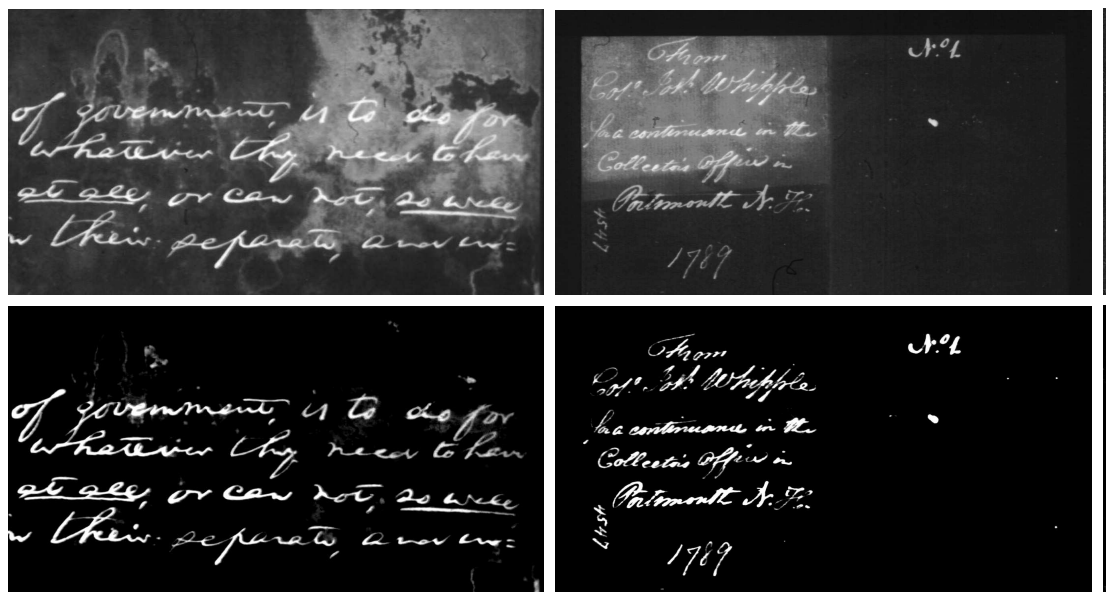
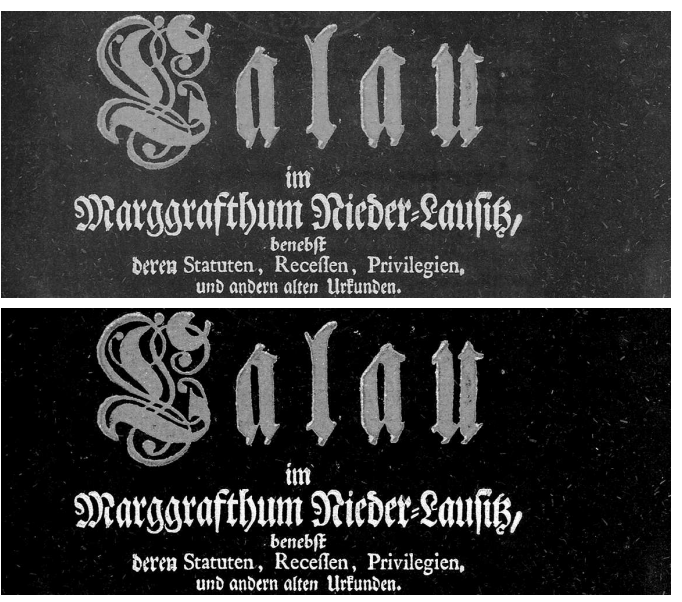

Figure 11. Background removal based on h-component tree processing: original document images (top) and corresponding results of the proposed background removal method (bottom).

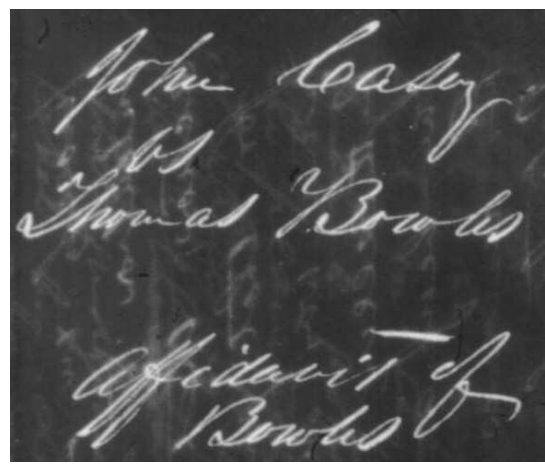

Der 9?atur, und Diefe fino Die Lranfange Der Pinge:

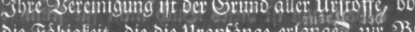

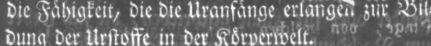
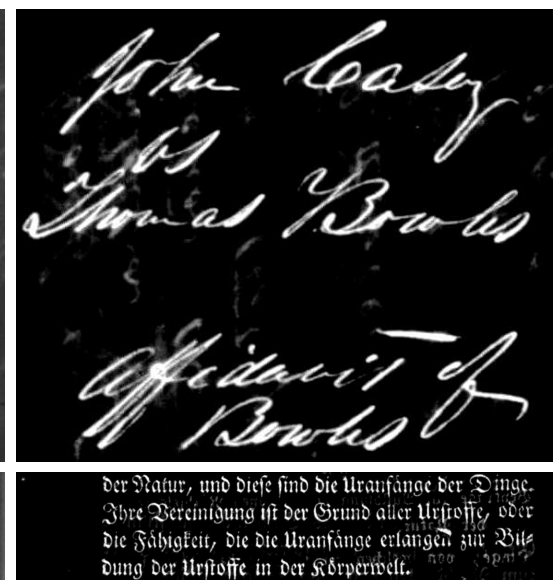

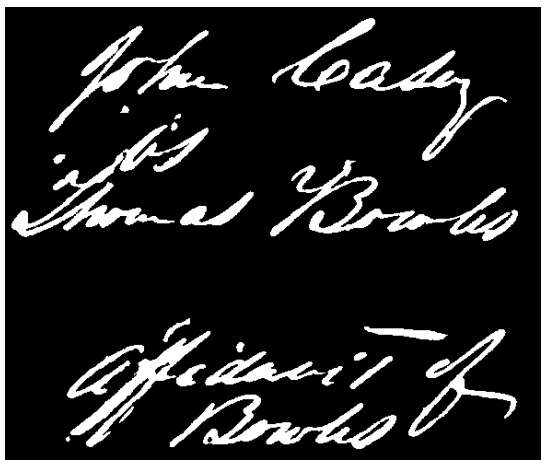

Der Platur, und diefe find ore Uranfinge Der Dinge.

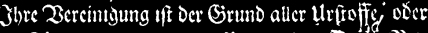

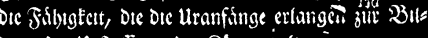
oung Der lirftofie in Der Sosperivelt.

Figure 12. Document image binarization using the proposed approach. Each line presents the processing of one document image from the DIBCO 2009 dataset [28]. From left to right: original image, image after background removal, and binarization result.

The notion of grayscale hierarchical representation is an emerging notion with several recent developments [22], [23], [24], [37], [38], and we believe that the $\mathrm{h}$-connection theory is a valuable framework for it. Our work provides a solution to combine the theory of hconnections with a hierarchical representation.

There are a lot of perspectives to this work. From a theoretical point of view, our work on the h-connection axiomatic may be considered as the beginning of the exploration of the zoology of h-connections. It seems clear that that large groups of h-connections must exist which share similar properties of their invariances, the behavior of their z-zones (do they fulfill the requirements of proposition 7 ?). Identifying these groups will certainly benefit most of the work based on h-connections.

From a more practical point of view, there is actually no general algorithm to build the h-component tree. It would be interesting to determine if the h-component tree of a group of h-connections could be built using a general algorithm algorithm as is possible to do for the mask based second generation connections.

\section{APPENDIX ProOFs}

Proposition 1: Let $a$ in $\mathcal{L},\left\{h_{i}\right\} \subseteq \gamma^{*}(a)$ and $b=$ $\bigvee\left\{h_{i}\right\}$, for all $i$, we have $h_{i} \leq b$ and $b \leq a$. Suppose that there exists some $h \in \mathcal{C}^{+}$such that $h_{i} \leq h \leq b \leq a$. But by definition of $\gamma^{*}(a)$ and as $h_{i}$ belongs to $\gamma^{*}(a)$, we have $h_{i}=h$ showing that $h_{i} \in \gamma^{*}(b)$. Thus we have $\left\{h_{i}\right\} \subseteq \gamma^{*}\left(\bigvee\left\{h_{i}\right\}\right)$.

Proposition 2: Let $s$ in $\mathcal{S}, h \in \mathcal{C}^{+}$such that $\gamma_{s}(a)=h$ and $h^{\prime} \in \gamma^{*}(a)$ such that $s \leq h^{\prime}$. We have $h^{\prime} \leq$ $\bigvee\left\{g \in \gamma^{*}(a) \mid s \leq g\right\}=h$, so $s \leq h^{\prime} \leq h \leq a$ and by definition of h-components $h=h^{\prime}$.

Proposition 3: We first show that $\mathrm{P}-2 \Rightarrow \mathrm{P}-1$. Let $\left\{h_{i}\right\} \subseteq \gamma^{*}(a)$, and assume that $\exists h^{\prime} \in\left\{h_{i}\right\}$ such that $\bigvee\left\{h \in \gamma^{*}(a) \mid h \neq h^{\prime}\right\}=\bigvee \gamma^{*}(a)$. Then, let $F=$ $\bigvee\left\{h \in \gamma^{*}(a) \mid h \neq h^{\prime}\right\}$, one have $h^{\prime} \leq \bigvee \gamma^{*}(a)=\bigvee F$ but that contradicts P-2 since $h^{\prime} \notin F$.

Then, we show that P-1 $\Rightarrow \mathrm{P}-2$ : let $\left\{h_{i}\right\} \subset \gamma^{*}(a)$ and assume that there exists $h \in \gamma^{*}(a)$ such that $h \notin\left\{h_{i}\right\}$ and $h \leq \bigvee\left\{h_{i}\right\}$. Let $b=\bigvee\left\{h_{i}\right\}=\bigvee\left(\left\{h_{i}\right\} \cup\{h\}\right)$, we have by 
proposition 1 that $h \in \gamma^{*}(b)$ and that $\left\{h_{i}\right\} \subseteq \gamma^{*}(b)$. And then we have $\bigvee\left\{h^{\prime} \in \gamma^{*}(b) \mid h^{\prime} \neq h\right\}=\bigvee\left\{h_{i}\right\}=b$ which contradicts $\mathrm{P}-1$.

Now P-3 $\Rightarrow$ P-2: let $\left\{h_{i}\right\} \subseteq \gamma^{*}(a)$ and $h \in \gamma^{*}(a)$. Assume that $h \leq \bigvee\left\{h_{i}\right\}$ then, by P-3, there exists $s \in \mathcal{S}$ such that $\gamma_{s}(a)=h$ and $s \leq h \leq \bigvee\left\{h_{i}\right\}$. By assumption, the family $\gamma^{*}(a)$ is finite, so there exists $j$ such that $s \leq h_{j}$ and from proposition 2 we can say that $h=h_{j}$ and thus $h$ belongs to $\left\{h_{i}\right\}$.

And finally, $\mathrm{P}-1 \Rightarrow \mathrm{P}-3$ : let $h \in \gamma^{*}(a), \mathrm{P}-1$ implies that $\bigvee B<a$ with $B=\left\{h^{\prime} \in \gamma^{*}(a) \mid h^{\prime} \neq h\right\}$. Now, consider the smallest family $\left\{s_{i}\right\} \subseteq \mathcal{S}$ such that $\bigvee\left(B \cup\left\{s_{i}\right\}\right)=a$. Let $s$ be an element of $\left\{s_{i}\right\}$, we have for all $h^{\prime} \in B, s \not \leq h^{\prime}$ and $s \leq h$ thus $\gamma_{s}(a)=h$.

Proposition 4: We already have the first inclusion $\left\{h_{i}\right\} \subseteq \gamma^{*}\left(\bigvee\left\{h_{i}\right\}\right)$ by proposition 1 . The second inclusion $\gamma^{*}\left(\bigvee\left\{h_{i}\right\}\right) \subseteq\left\{h_{i}\right\}$ is directly given by P-2: let $h \in \gamma^{*}\left(\bigvee\left\{h_{i}\right\}\right)$ as we have $h \leq \bigvee\left\{h_{i}\right\}$, P-2 says that $h$ belongs to $\left\{h_{i}\right\}$. Finally, the double inclusion proves that $\left\{h_{i}\right\}=\gamma^{*}\left(\bigvee\left\{h_{i}\right\}\right)$.

Proposition 5: First, we show that $\forall s_{1}, s_{2} \in \mathcal{S}$, $s_{1} \stackrel{a_{*}}{\sim} s_{2} \Rightarrow s_{1} \stackrel{a}{\sim} s_{2}$, this part does not need the accessibility property. We have:

$$
\begin{aligned}
s_{1} \stackrel{a_{*}^{*}}{\sim} s_{2} & \Leftrightarrow \gamma_{s_{1}}^{*}(a)=\gamma_{s_{2}}^{*}(a) \\
& \Rightarrow \bigvee \gamma_{s_{1}}^{*}(a)=\bigvee \gamma_{s_{2}}^{*}(a) \\
& \Leftrightarrow \gamma_{s_{1}}(a)=\gamma_{s_{2}}(a) \\
& \Leftrightarrow s_{1} \stackrel{a}{\sim} s_{2}
\end{aligned}
$$

The reverse implication is given by: assume that we have $s_{1} \stackrel{a}{\sim} s_{2}$, which is equivalent to $\gamma_{s_{1}}(a)=\gamma_{s_{2}}(a)$. Let $h \in$ $\gamma_{s_{1}}^{*}(a)$, we have $h \leq \gamma_{s_{1}}(a)=\bigvee \gamma_{s_{1}}^{*}(a)=\bigvee \gamma_{s_{2}}^{*}(a)$. Then, P-2 implies that $h \in \gamma_{s_{2}}^{*}(a)$ and thus $\gamma_{s_{1}}^{*}(a) \subseteq \gamma_{s_{2}}^{*}(a)$. The same argument is used to prove that $\gamma_{s_{2}}^{*}(a) \subseteq \gamma_{s_{1}}^{*}(a)$ showing that $\gamma_{s_{2}}^{*}(a)=\gamma_{s_{1}}^{*}(a)$ which is equivalent to $s_{1} \stackrel{a_{*}}{\sim} s_{2}$.

Proposition 6: We have to show that $\preceq$ is a reflexive, anti-symmetric, and transitive relation on $\mathcal{I}$.

Reflexivity is trivial as $\forall x \in \mathcal{I}$, $\operatorname{supp}(x) \subseteq \operatorname{supp}(x)$ and $x / \operatorname{supp}(x)=x$ and thus $x \preceq x$.

We now show that the relation is anti-symmetric. Let $x, y$ be two elements of $\mathcal{I}$ such that $x \preceq y$ and $y \preceq x$. We have $\operatorname{supp}(x) \subseteq \operatorname{supp}(y)$ and $\operatorname{supp}(y) \subseteq \operatorname{supp}(x)$ and thus $\operatorname{supp}(y)=\operatorname{supp}(x)$. Then as the two functions have the same support: $x / \operatorname{supp}(y)=x$ and $y_{/ \operatorname{supp}(x)}=y$. So $x \leq y$ and $y \leq x$ implies $x=y$ as $\leq$ is anti-symmetric.

We now prove transitivity. Let $x, y$ and $z$ be three elements of $\mathcal{I}$ such that $x \preceq y$ and $y \preceq z$. As $\operatorname{supp}(x) \subseteq$ $\operatorname{supp}(y)$ and $\operatorname{supp}(y) \subseteq \operatorname{supp}(z)$, we have $\operatorname{supp}(x) \subseteq$ $\operatorname{supp}(z)$. And since $z / \operatorname{supp}(y) \leq y$ and $\operatorname{supp}(x) \subseteq \operatorname{supp}(y)$ then $z / \operatorname{supp}(x) \leq y / \operatorname{supp}(x)$, finally $z / \operatorname{supp}(z) \leq x$ and so $x \preceq z$.

Proposition 7: We show that for all $x \neq \perp$ in $G$ there exists at most one $y$ in $G$ such that $(x, y)$ is in $V$ (if $x=\perp$, $x$ is the least element and there cannot exist $y \in G$ such that $(x, y)$ is in $V)$. Assume that there exists $y$ and $y^{\prime}$ in $G, y \neq y^{\prime}$ such that $(x, y) \in V$ and $\left(x, y^{\prime}\right) \in V$. We have $x \preceq y$ and $x \preceq y^{\prime}$, so $\operatorname{supp}(x) \subseteq \operatorname{supp}(y)$ and $\operatorname{supp}(x) \subseteq$ $\operatorname{supp}\left(y^{\prime}\right)$, thus $\operatorname{supp}(y) \cap \operatorname{supp}\left(y^{\prime}\right) \supseteq \operatorname{supp}(x) \neq \emptyset$ and thus $y \wedge \preceq y^{\prime} \neq \perp$. By hypothesis, as $y$ and $y^{\prime}$ are not disjoint they are comparable: $y \preceq y^{\prime}$ or $y^{\prime} \preceq y$. First case, assume that $y \preceq y^{\prime}$, then $\left(x, y^{\prime}\right)$ cannot be in $V$ as we have $x \preceq y \preceq y^{\prime}$. The second case is similar which contradicts the assumption. As each node of $G$ can have at most one antecedent the graph is acyclic (other cycles are not possible due to the ordering relation).

\section{REFERENCES}

[1] J. Serra, Image Analysis and Mathematical Morphology. II: Theoretical Advances. London, UK: Academic Press, 1988.

[2] H. Heijmans, "Connected morphological operators for binary images," Computer Vision and Image Understanding, vol. 73, no. 1, pp. 99-120, 1999.

[3] J. Serra and P. Salembier, "Connected operators and pyramids," in Image Algebra and Morphological Image Processing IV, E. R. Dougherty, P. D. Gader, and J. C. Serra, Eds., vol. 2030, no. 1. SPIE, 1993, pp. 65-76.

[4] P. Salembier and J. Serra, "Flat zones filtering, connected operators, and filters by reconstruction," IEEE Transactions on Image Processing, vol. 4, no. 8, pp. 1153-1160, 1995.

[5] P. Salembier, A. Oliveras, and L. Garrido, "Anti-extensive connected operators for image and sequence processing," IEEE Transactions on Image Processing, vol. 7, no. 4, pp. 555-570, 1998.

[6] F. Cheng and A. Venetsanopoulos, "An adaptive morphological filter for image processing," IEEE Transactions on Image Processing, vol. 1, no. 4, pp. 533-539, 1992.

[7] E. Breen and R. Jones, "Attribute openings, thinnings, and granulometries," Computer Vision and Image Understanding, vol. 64, no. 3, pp. 377-389, 1996.

[8] R. Jones, "Connected filtering and segmentation using component trees," Computer Vision and Image Understanding, vol. 75, no. 3, pp. 215-228, 1999.

[9] D. Gatica Perez, C. Gu, M. Sun, and S. Ruiz Correa, "Extensive partition operators, gray-level connected operators, and region merging/classification segmentation algorithms: theoretical links," IEEE Transactions on Image Processing, vol. 10, no. 9, pp. 1332-1345, 2001.

[10] J. Serra, "A lattice approach to image segmentation," Journal of Mathematical Imaging and Vision, vol. 24, no. 1, pp. 83-130, 2006.

[11] B. Naegel, N. Passat, N. Boch, and M. Kocher, "Segmentation using vector-attribute filters: methodology and application to dermatological imaging," in International Symposium on Mathematical Morphology, G. Bannon, J. Barrera, and U. Braga-Neto, Eds., vol. 1. Rio de Janeiro, Brazil: INPE, 2007, pp. 239-250.

[12] C. Berger, T. Geraud, R. Levillain, N. Widynski, A. Baillard, and E. Bertin, "Effective component tree computation with application to pattern recognition in astronomical imaging," in IEEE International Conference on Image Processing, vol. 4, 2007, pp. 41-44.

[13] L. Najman and M. Couprie, "Building the component tree in quasi-linear time," IEEE Transactions on Image Processing, vol. 15, no. 11, pp. 3531-3539, 2006.

[14] L. Chen, M. Berry, and W. Hargrove, "Using dendronal signatures for feature extraction and retrieval," International Journal of Imaging Systems and Technology, vol. 11, no. 4, pp. 243-253, 2001.

[15] J. Mattes and J. Demongeot, "Efficient algorithms to implement the confinement tree," in International Conference on Discrete Geometry for Computer Imagery, London, UK, 2000, pp. 392-405.

[16] C. Ronse, "Partial partitions, partial connections and connective segmentation," Journal of Mathematical Imaging and Vision, vol. 32, no. 2, pp. 97-125, 2008.

[17] U. Braga-Neto and J. Goutsias, "Connectivity on complete lattices: New results," Computer Vision and Image Understanding, vol. 85, no. 1, pp. 22-53, 2002.

[18] G. Ouzounis and M. Wilkinson, "Mask-based second-generation connectivity and attribute filters," IEEE Transactions on Pattern Analysis and Machine Intelligence, vol. 29, no. 6, pp. 990-1004, 2007.

[19] J. Serra, "Connectivity on complete lattices," Journal of Mathematical Imaging and Vision, vol. 9, no. 3, pp. 231-251, 1998. 
[20] U. Braga-Neto and J. Goutsias, "A theoretical tour of connectivity in image processing and analysis," Journal of Mathematical Imaging and Vision, vol. 19, no. 1, pp. 5-31, 2003.

[21] U. Braga-Neto and J. Goutsias, "Grayscale Level Connectivity: Theory and Applications," IEEE Transactions on Image Processing, vol. 13, pp. 1567-1580, 2004.

[22] O. Nempont, J. Atif, E. Angelini, and I. Bloch, "A new fuzzy connectivity measure for fuzzy sets," Journal of Mathematical Imaging and Vision, vol. 34, no. 2, pp. 107-136, 2009.

[23] G. Ouzounis, "Generalized connected morphological operators for robust shape extraction," Ph.D. dissertation, University of Groningen, 2009.

[24] G. Ouzounis and M. Wilkinson, "Hyperconnected attribute filters based on k-flat zones," IEEE Transactions on Pattern Analysis and Machine Intelligence, vol. accepted, 2010.

[25] M. Wilkinson, "An axiomatic approach to hyperconnectivity," in Mathematical Morphology and Its Application to Signal and Image Processing, 9th International Symposium, ISMM, ser. LNCS, vol. 5720, 2009, pp. 35-46.

[26] _ "Hyperconnectivity, attribute-space connectivity and path openings: Theoretical relationships," in Mathematical Morphology and Its Application to Signal and Image Processing, 9th International Symposium, ISMM, ser. LNCS, vol. 5720, 2009, pp. 47-58.

[27] P. Salembier and M. Wilkinson, "Connected operators," IEEE Signal Processing Magazine, vol. 26, no. 6, pp. 136-157, 2009.

[28] B. Gatos, K. Ntirogianni, and I. Pratikakis, "ICDAR 2009 document image binarization contest (DIBCO)," in International Conference on Document Analysis and Recognition, 2009, pp. 1375-1382.

[29] G. Grätzer, General Lattice Theory. Birkhauser, 2007.

[30] H. Heijmans, Morphological Image Operators. Boston, US: Academic Press, 1994.

[31] U. Braga-Neto and J. Goutsias, "A multiscale approach to connectivity," Computer Vision and Image Understanding, vol. 89, no. 1, pp. 70-107, 2003.

[32] A. Rosenfeld, "Fuzzy digital topology," Information and Control, vol. 40, no. 1, pp. 76-87, 1979.

[33] M. Grimaud, "New measure of contrast: the dynamics," in Image Algebra and Morphological Image Processing III, P. Gader, E. Dougherty, and J. Serra, Eds. SPIE, 1992, pp. 292-305.

[34] E. Aptoula and S. Lefèvre, "A comparative study on multivariate mathematical morphology," Pattern Recognition, vol. 40, no. 11, pp. 2914-2929, 2007.

[35] B. Naegel and N. Passat, "Component-trees and multi-value images: A comparative study," in athematical Morphology and Its Application to Signal and Image Processing, 9th International Symposium, ISMM, ser. LNCS, vol. 5720, 2009, pp. 261-271.

[36] I. Pratikakis, B. Gatos, and K. Ntirogianni, "H-dibco 2010 handwritten document image binarization competition," in International Conference on Frontiers in Handwriting Recognition, 2010.

[37] P. Soille, "Constrained connectivity for hierarchical image partitioning and simplification," IEEE Transactions on Pattern Analysis and Machine Intelligence, vol. 30, no. 7, pp. 1132-1145, 2008.

[38] G. Palma, I. Bloch, and S. Muller, "Fast fuzzy connected filter implementation using max-tree updates," Fuzzy Sets and Systems, vol. 161, no. 1, pp. 118-146, 2010.

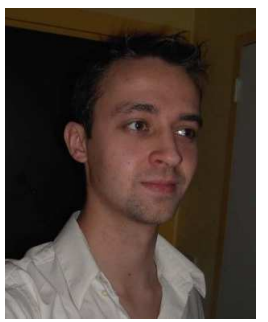

Benjamin Perret received his M.Sc. in Computer Science in 2007, and his Ph.D. in Image Processing in 2010 from the University of Strasbourg, France. He currently helds an Assistant Professor position at the University of Strasbourg in the Image Science, Computer Science and Remote Sensing Laboratory. His Ph.D. aims at developing new methods for the automatic spectro-morphological characterization of multispectral galaxy observations. The challenge consists in fitting galaxy models and multiband observations to classify each galaxy according to its spectral behavior and its morphological features in an unsupervised way. His research interests are in mathematical morphology (theory of connections, hierarchical representations and multispectral image processing), Bayesian modeling and Markov chains Monte Carlo optimization methods.

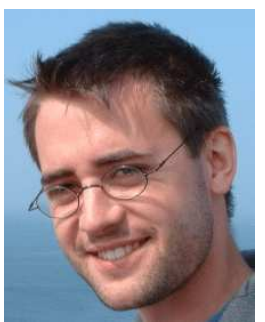

Sébastien Lefèvre received his M.Sc. and Eng. degrees in Computer Engineering from the University of Technology of Compiègne, and his Ph.D. in Computer Science from the University of Tours in 2002. In 2009, he earned his French HDR degree in Computer Science from the University of Strasbourg. From 2003 to 2010, he was an Associate Professor in the Department of Computer Sciences and the Image Sciences, Computer Sciences and Remote Sensing Laboratory (LSIIT), University of Strasbourg - CNRS. In 2009-2010, he was an INRIA invited scientist within the TEXMEX team of IRISA/INRIA Rennes. In 2010, he joined the University of South-Brittany as a Full Professor in Computer Science, in the Institute of Technology of Vannes and the VALORIA research laboratory. His research interests are in image analysis and pattern recognition, using mainly mathematical morphology and machine learning techniques with applications in space and earth observation and content-based image/video retrieval.

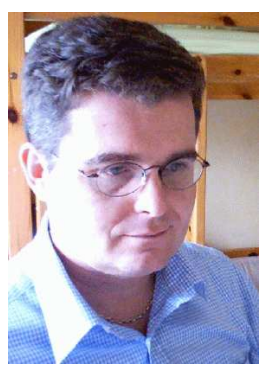

Christophe Collet was born in 1966 in France. He graduated from the Université Paris-Sud Orsay in 1989 (Master in Signal Processing, DEA) and received a Ph.D. in Image Processing from the University of Toulon in 1992. He spent 8 years at the French Naval Academy where he created and led the laboratory GTS 'Groupe de Traitement du Signal' from 1994 to 2000. During these years, he developed hierarchical Markovian approaches for SONAR image analysis and remote sensing multiband image reconstruction. Since 2001, he has held a full Professor position at the University of Strasbourg where he joined the Image Sciences, Computer Sciences and Remote Sensing Laboratory (LSIIT UMR CNRS 7005). From this date, he has developed new statistical image analysis both for medical and astronomy applications. Independently of the application, he develops new approaches based on Bayesian inference and graphical models for image segmentation, classification, fusion or restoration. He currently works on fusion of massive hyperspectral data and unsupervised multiband galaxy classification in astronomy, segmentation of brain MRI, detection of lesions on multimodal $3 d+t$ MRI with Strasbourg Hospital physicians. His major research interests include multi-image segmentation and classification with hierarchical approaches (wavelet decomposition, multigrid optimization, multiscale modeling), Bayesian inference, Markovian chain/field/tree modeling, approaches for pattern recognition, Bayesian networks, with a particular focus on astronomy (hyperspectral) and medical (multimodal) images since 2001.

Eric Slezak was born in 1961 in France. He graduated from the Universite Nice - Sophia Antipolis in 1984 (Master in Signal Processing, DEA) and received a Ph.D. in Physics from the same university in 1988. His thesis research was carried out at the Nice Astronomical Observatory and dealt with the development of algorithms for object detection and classification in wide-field optical images. He was among the first to introduce multiscale techniques in observational cosmology, developing wavelet-based approaches to analyze images, describe complex objects, compute density probability functions, perform spectro-imagery from low S/N X-ray data. Doing so, he contributed in quantifying the subclustering, segregation properties and dynamical status of several clusters of galaxies and in detecting large scale diffuse emission related to their building histories. Since 2006, he has held a full astronomer position at the Observatoire de la Cote d'Azur. His research interests include astronomical image segmentation and classification from multiwavelength data analysis. Since 2009 , he has been leading a 4-year IT research project aiming to develop the signal processing methods required to analyze the massive hyperspectral datasets provided by the forth-coming integral field spectrographs in astronomy. 